University of Louisville

ThinkIR: The University of Louisville's Institutional Repository

Electronic Theses and Dissertations

8-2007

\title{
Elastomer degradation in water utility systems via loss of carbon black observed with atomic force microscopy.
}

Randolph Norman Schoenbaechler 1972-

University of Louisville

Follow this and additional works at: https://ir.library.louisville.edu/etd

\section{Recommended Citation}

Schoenbaechler, Randolph Norman 1972-, "Elastomer degradation in water utility systems via loss of carbon black observed with atomic force microscopy." (2007). Electronic Theses and Dissertations. Paper 1279.

https://doi.org/10.18297/etd/1279

This Master's Thesis is brought to you for free and open access by ThinkIR: The University of Louisville's Institutional Repository. It has been accepted for inclusion in Electronic Theses and Dissertations by an authorized administrator of ThinkIR: The University of Louisville's Institutional Repository. This title appears here courtesy of the author, who has retained all other copyrights. For more information, please contact thinkir@louisville.edu. 
ELASTOMER DEGRADATION IN WATER UTILITY SYSTEMS VIA LOSS OF CARBON BLACK OBSERVED WITH ATOMIC FORCE MICROSCOPY

By

Randolph Norman Schoenbaechler

B.S. in Chemical Engineering, University of Louisville, 2005

\author{
A Thesis \\ Submitted to the Faculty of the \\ University of Louisville \\ J.B. Speed School of Engineering \\ in Partial Fulfillment of the Requirements \\ for the Professional Degree \\ MASTER OF ENGINEERING
}

Department of Chemical Engineering

August 2007 
ELASTOMER DEGRADATION IN WATER UTILITY SYSTEMS VIA LOSS OF CARBON BLACK OBSERVED WITH ATOMIC FORCE MICROSCOPY

Submitted by:

Randolph Norman Schoenbaechler

A Thesis Approved on

(Date)

by the Following Reading Committee:

Gerold A. Willing, Thesis Director

James C. Watters

Thomas D. Rockaway 


\section{ACKNOWLEDGEMENTS}

I would like to sincerely thank my academic director, Dr. Gerold Willing, for his guidance throughout my graduate study and thesis work. I would like to express my gratitude to Mr. Mark Schreck for his support and encouragement. I feel very appreciative for the opportunity provided by Dr. Thomas Rockaway to participate in this study. I would also like to thank Ms. Rodica McCoy for help with learning to properly use some of the instrumentation necessary for completion of this work. I would like very much to thank Dr. Jim Watters for his support and advice throughout my academic studies. His faith in my abilities as an engineer has provided me with opportunities to achieve my goals in life. I would also like to thank my parents and sister for expecting me to achieve my goals. 


\begin{abstract}
The use of monochloramines in the potable water supply industry has been selected as the disinfectant method of choice due to a longer term of effectiveness and less toxic by-products than the previous choice of chlorine. In spite of the benefits exhibited by the use of monochloramines much greater rates of elastomer degradation have been noted in the water industry. The modes of attack by monochloramines on the rubber parts are still poorly understood.
\end{abstract}

A common ingredient in nearly all elastomer formulations is carbon black. In addition to its role as a filler material, carbon black is also known to add strength and durability to the elastomer. Degradation of average particle size showing that carbon black particles dissolve into the invasive liquid medium can be observed using phase images produced by atomic force microscopy. The nanoscale size of the carbon black particles makes the use of the AFM an ideal investigative tool. Elastomeric properties of the rubber coupons exhibit similar trends to the degradation of the carbon black material in the polymer matrix. Diffusion coefficients approximated by use of a simplified form of Fick's second law also shows a similar trend. Use of monochloramines as a disinfectant by the water supply industry produce fewer toxic by-products and maintain a longer useful potable water life than chlorine; however, rapid degradation of rubber parts will continue unless more suitable formulations can be found. 


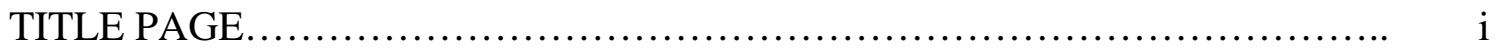

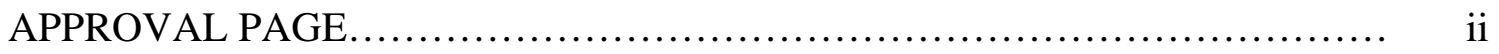

ACKNOWLEDGEMENTS ................................................ iii

ABSTRACT ..................................................................... iv

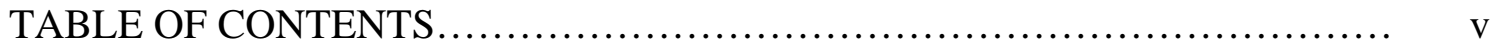

LIST OF TABLES.......................................................... vi

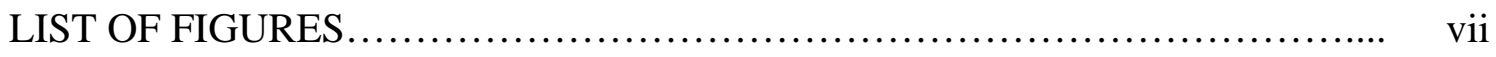

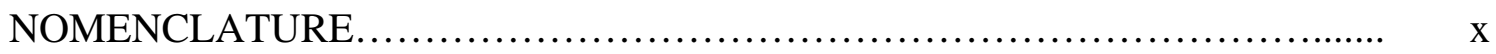

I. INTRODUCTION................................................. 1

IA. Water Supply Disinfection........................................ 1

IB. Reinforcement of Elastomers.......................................... 2

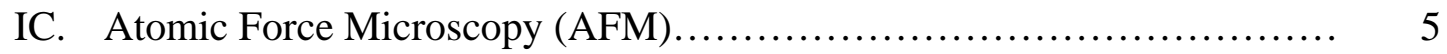

ID. Overview of Experimental Approach................................... 7

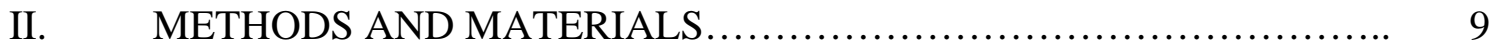

IIA. Aging and Determination of Effects on Bulk Properties.................... 9

IIB. Cross Sectioning and Imaging with Atomic Force Microscopy............. 16

III. RESULTS AND DISCUSSION OF RESULTS.......................... 23

IIIA. Introduction........................................................ 23

IIIB. Testing of Elastomeric Properties................................. 30

IIIC. Atomic Force Microscopy Data...................................... 35 
IIID. Estimated Diffusion Coeficients.................................. 59

IV. CONCLUSIONS AND RECOMMENDATIONS...................... 66

REFERENCES CITED.............................................. 70

VITA............................................................... 72 


\section{LIST OF TABLES}

TABLE

PAGE

2.1 Natural Rubber Formulation........................................ 11

2.2 Styrene Butadiene Rubber Formulation............................... 12

2.3 EPDM-S Rubber Formulation.......................................... 12

3.1 Diffusion coefficients determined after accelerated aging for 30 days......... 64

3.2 Comparison of estimated diffusion coefficients with monochloramines........ 65 


\section{LIST OF FIGURES}

1.1 Molecular structure of elastomers...................................... 4

1.2 Concept and major components of AFM.................................. 6

2.4 Rubber slab, die and press (left), samples cut

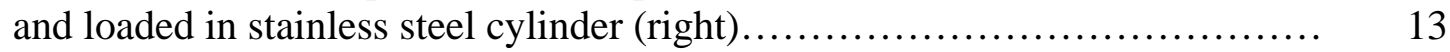

2.5 Water bath (left), titrimeter in center and $\mathrm{pH}$ meter (right).................. 15

2.6 Analytical balance with water submersion

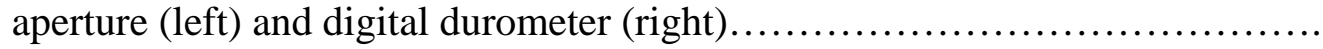

2.7 Full sized rubber sample next to sample broken using liquid nitrogen and cross sectioned sample cut from near center appropriate for AFM imaging.

3.2 Cross section of Natural Rubber first and third rows, greater magnified pictographs of area corresponding to middle of region located directly above second and fourth rows.

3.3 Cross section of SBR first and third rows, greater magnified pictographs of area corresponding to middle of region located directly above second and fourth rows.

3.4 Cross section of EPDM-S first and third rows, greater magnified pictographs of area corresponding to middle of region located directly above second and fourth rows.

3.5 Effects of Monochloramines and Chlorine in water on elastomeric properties of Natural Rubber.

3.6 Effects of Monochloramines and Chlorine in water on elastomeric properties of SBR.

3.7 Effects of Monochloramines and Chlorine in water on elastomeric properties of EPDM-S. 
3.9 Area fractions of Natural Rubber cross sections aged in water mixture with monochloramines.

3.10 Particle diameter in Natural Rubber cross sections aged in water mixture with monochloramines. ...

3.11 AFM images of Natural Rubber depicting effects of aging at the outer edge (left) and near the center (right) after 30 days aging with monochloramines...

3.12 Area fractions of SBR cross sections aged in water mixture with monochloramines.

3.13 Particle diameter in SBR cross sections aged in water mixture with monochloramines....

3.14 AFM images of SBR depicting near edge effects at 0 days (left) and after 30 days aging with monochloramines (right)...

3.15 Area fractions of EPDM-S cross sections aged in water mixture with monochloramines

3.16 Particle diameter in EPDM-S cross sections aged in water mixture with monochloramines

3.17 AFM images of EPDM-S depicting effect of aging at the outer edge (left) and near the center (right) after 30 days aging with monochloramines ...

3.18 Area fractions of Natural Rubber cross sections aged in water mixture with monochloramines and with chlorine.

3.19 Particle diameter in Natural Rubber cross sections aged in water mixture with monochloramines and with chlorine

3.20 Area fractions of SBR cross sections aged in water mixture with monochloramines and with chlorine

3.21 Particle diameter in SBR cross sections aged in water mixture with monochloramines and with chlorine

3.22 Area fractions of EPDM-S cross sections aged in water mixture with monochloramines and with chlorine

3.23 Particle diameter in EPDM-S cross sections aged in water mixture with monochloramines and with chlorine. 
3.24 AFM images of Natural Rubber depicting effect of aging at the outer edge (left) and near the center (right) after 30 days with chlorine....................

3.25 Sample Cross Sections at 0 days (left), 30 days in chlorine mixture (center) and monochloramines mixture (right) of each individual micrograph with Natural Rubber shown first, SBR second and EPDM-S third.............

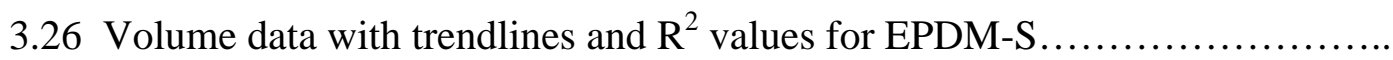




\section{NOMENCLATURE}
$\mathrm{A}=$ area of thin slice
$\mathrm{C}=$ concentration
$\mathrm{C}_{0}=$ initial bulk concentration
$\mathrm{C}_{\mathrm{S}}=$ concentration at surface
$\mathrm{D}=$ diffusion coefficient
$\mathrm{J}=$ flux
$\mathrm{J}_{\text {in }}=$ flux into differential volume
$\mathrm{J}_{\text {out }}=$ flux out of differential volume
$\mathrm{t}=$ time
$\mathrm{V}=$ volume of thin slice
$\mathrm{x}=$ penetration distance
$\mathrm{x}_{\mathrm{eff}}=$ effective penetration distance 


\section{INTRODUCTION}

\section{A. Water Supply Disinfection}

Municipal water facilities of the United States have included disinfectants in their purification process since the early 1900s. Free chlorine was the original disinfectant used throughout the industry because chlorine was known to be useful for the suppression of biological organisms. Regulatory and health issues have resulted in many potable water supply systems switching to monochloramines. Monochloramines are molecules which have an amine group attached to the chlorine molecule by reaction with ammonia. Monochloramines are generally preferred because of a longer term of effectiveness and tendency to form fewer toxic by-products. The use of monochloramines has unfortunately had significant negative impacts on the water distribution infrastructure occurring mostly in seemingly distinct regions around the country. While both free chlorine and monochloramines contribute to degradation of elastomers in the water systems' infrastructure, monochloramines appear to attack elastomer components much

more aggressively. Degradation of elastomers exposed to treated water depends on a number of poorly understood factors such as temperature of the system and concentration of disinfectant. Each elastomer formulation exhibits a unique reaction when it is exposed 
to free chlorine or monochloramines as some formulations have been observed to resist degradation while others degrade rapidly. The American Water Works Research Foundation has continued to investigate elastomer reactions to the disinfectants to which they are exposed. In recent AWWA projects, long term performance has been modeled and the rate of degradation predicted by accelerating degradation by varying temperature and concentration.

\section{B. Reinforcement of Elastomers}

Carbon black is widely used as reinforcing filler in nearly all elastomer compounds. The reinforcing properties of carbon black on the soft polymer matrix have been thoroughly investigated and are determined to be both physical and chemical in nature. It is widely accepted that voids caused by loss of carbon black from the matrix allows propagation of cracks extending inward from the surface (Schaffer et al., 1995). In the presence of a relatively hard carbon black particle, crack propagation may be stopped. This is due to the hard physical nature of the particle and the energy dispersing effect on the leading edge of the fissure due to molecular forces of attraction at the interface of the carbon black surface and the surrounding polymer matrix. Voids that have a diameter on the order of tens of nanometers have little energy dissipating effects and may actually enable propagation. (Willing, 2007) Additionally, carbon black helps prevent decay of elastomers by allowing molecular slippage including molecular bond rupture and reformation between the carbon black particle and the adjacent polymer 
chains during conditions of strain. This allows for stress distribution and equalization (Donnet et al., 1976).

This report describes a series of experimental techniques, results and conclusions from investigations into the effects that water disinfected with monochloramines, and conversely with chlorine, has on the degradation of carbon black, and the nature of this effect. The three types of rubber selected for this study are Natural Rubber, Styrene Butadiene Rubber (SBR) and Ethylene Propylene Diene Monomer sulfur cured (EPDMS). The types of rubber were selected based on their performance and prevalence in the water industry. In this and in earlier studies it has been found that of the three types of rubber selected Natural Rubber performs the most poorly, with SBR performing slightly better and EPDM retaining its physical qualities substantially better than either. This may be due to the molecular structure of the backbone material in EPDM.

Natural Rubber and SBR are very similar only differing by the presence of styrene containing a benzene ring structure occurring in one out of every two or three repeat units in SBR in place of a simple methane group as found in Natural Rubber. The polymer chains of EPDM have a very different structure. The three polymer structures are shown in Figure 1.1.

The focus of the experiment is to show empirically that the carbon black particles are being reduced in both size and concentration within the elastomer sample, and how deeply within the sample these effects penetrate over time correlated with bulk scale measurements. The purpose of this is to show that monochloramines attack the polymers in part by wearing away the carbon black embedded in its matrix. Techniques of Scanning Electron Microscopy, SEM, are also used in imaging and determination of the 
effects on carbon black embedded in the polymer matrix. A diffusion coefficient for disinfectant solution using chlorine and solution using monochloramines based on Fick's second law is also be estimated using optical micrograph data.

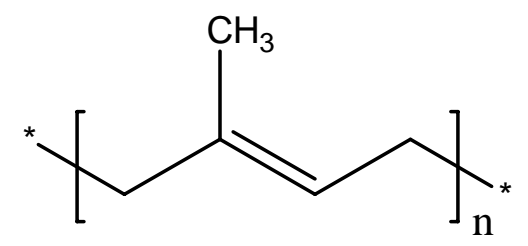

Natural Rubber (NR)

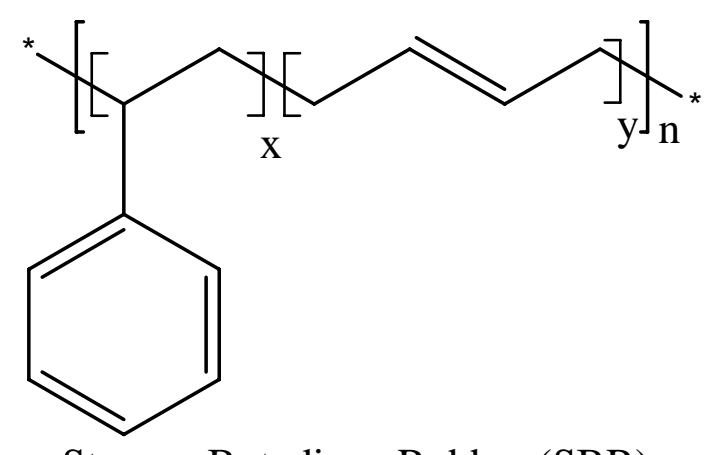

Styrene Butadiene Rubber (SBR)

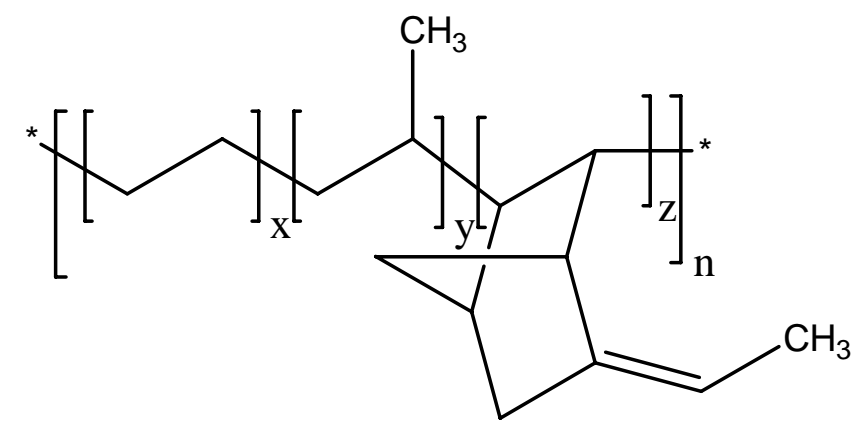

Ethylene Propylene Diene Monomer (EPDM)

Figure 1.1 Molecular structure of elastomers 


\section{Atomic Force Microscopy (AFM)}

Invented by Gerd Binnig, Calvin Quate and Christoph Gerber in 1986 (Binnig et al., 1986), Atomic Force Microscopy has found many uses in scientific and analytical fields due mainly to its ability to measure three dimensional images with high nanoscale resolution in a variety of applications such as quality control and product development (Rugar et al., 1990). Researchers have employed the AFM to visualize and measure the dimensions of molecules on surfaces (Yang et al. 1992), as well as to determine characteristics and properties of objects on the nanometer scale. It became apparent that the AFM was also capable of leaving a mark, or an impression on certain surfaces, as the scanning probe passed over the sample. The ability to coat the tip of the probe with charged particles that could be deposited much like ink on a page was also developed (Kwak et al., 2004). The AFM is also an important tool for inspecting optical disk stampers, measuring line widths on integrated circuit masks, and for visualizing thin film growth morphology and grain size (Ruger et al., 1990). The ability of the AFM to visualize and help to determine grain size is important to the work presented in this study where carbon black particles were imaged and sized.

The AFM scans over a sample surface using a fine $\mathrm{Si} / \mathrm{SiO}_{2}$ tip. The tip is located at the end of a cantilever with a laser beam positioned near the end of cantilever on the opposite side as the tip. As the tip is either repelled by or attracted to the surface, the cantilever deflects; this results in a changing of the laser beam position as it is reflected 
off the cantilever onto a photodiode receptor. The photodiode detects the relative position of the laser on the photodiode face as shown in Figure 1.2. A plot of the laser deflection versus tip position in the $\mathrm{x}, \mathrm{y}$ and $\mathrm{z}$ directions on the sample surface provide 2 or 3 dimensional surface images. A sensitive piezoelectric element controls the position and motion of the tip on a surface. Normally, a piezoelectric element can be divided into three sections responsible for the $\mathrm{x}, \mathrm{y}$ and $\mathrm{z}$ motion as shown in Figure 1.2.

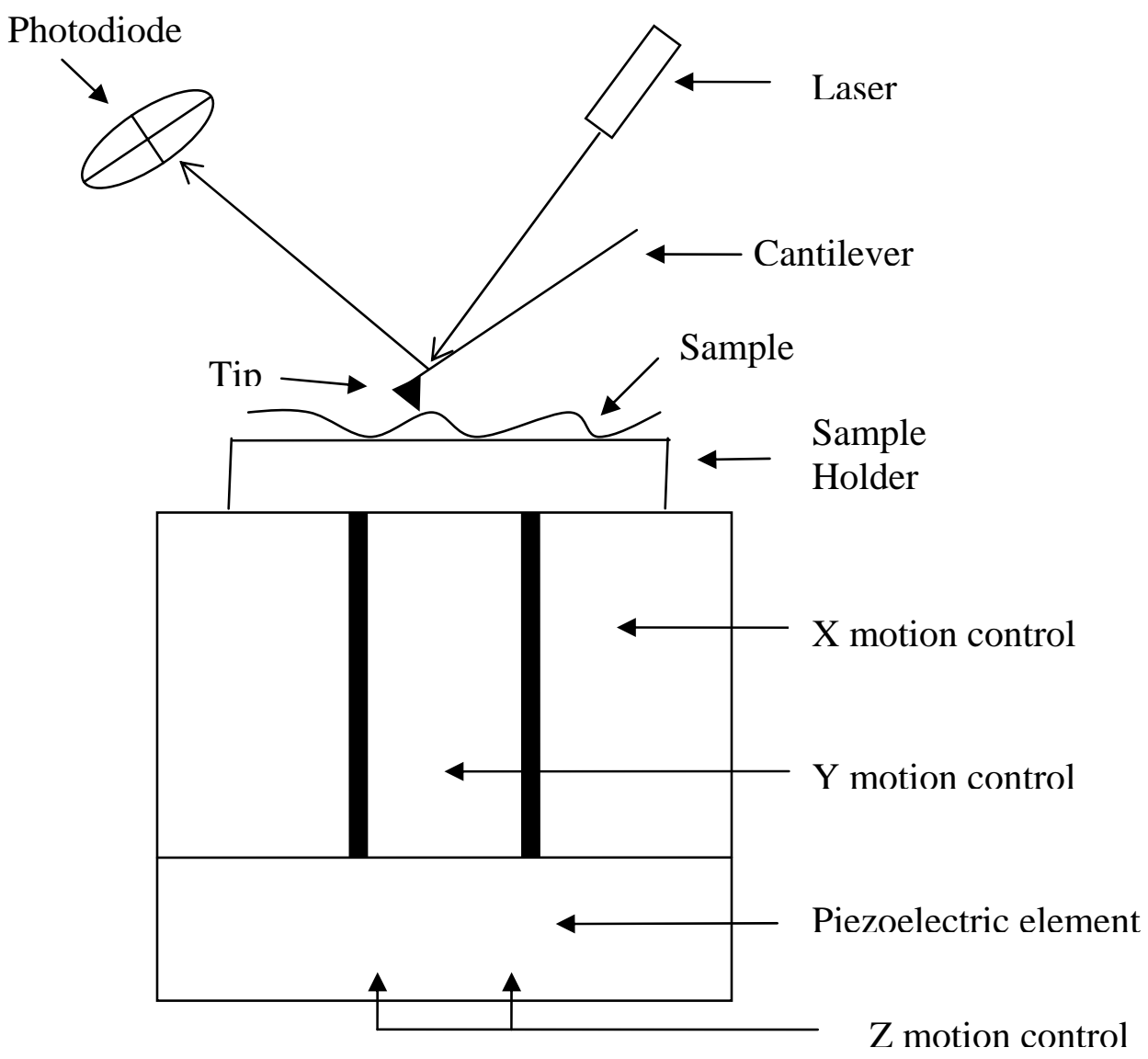

Figure 1.2 Concept and major components of AFM

The three major modes of AFM operation are contact, non-contact and tapping mode. When originally developed, the AFM was only capable of operating in contact mode (Binnig et al., 1986). In this mode the tip is placed in direct contact with the 
sample surface. The deflection of the cantilever is generated by variations of the surface topography. Normally, contact mode can be performed under air, liquid or vacuum conditions. In the case of soft surfaces such as in polymers, which are easily damaged, contact mode might not be the best technique to use due to tip-induced sample damage. Advancements in AFM technology now allow the user to produce images without contacting the surface. In non-contact mode, first introduced by Martin and coworkers (Martin et al. 1987), measurements of forces acting between the tip and a sample surface are used to produce the images. A tip to sample distance of about $30-150 \AA$ is maintained. Long-range molecular forces such as Van der Waals forces induce deflections in the cantilever and allow for amplitude, frequency, phase and topographic images to be constructed (Martin et al., 1987). The third technique is tapping mode and is sometimes referred to as intermittent-contact, or more generally, as Dynamic Force Mode (DFM). In tapping mode the cantilever is oscillated at its resonance frequency, and is only in contact with the surface for a fraction of its oscillation period. Phase imaging was introduced as an off-shoot of tapping mode (Chernoff et al., 1995). Phase images are produced by measuring the difference between the oscillations of the cantilever driving piezo and the oscillations detected by the photodiode. It is thought that image contrast is derived from the surface properties such as stiffness and viscoelasticity.

\section{Overview of Experimental Approach}

Rectangular elastomer coupons were cut with a die and press from rubber slabs provided by Ashtabula Rubber. The samples were subjected to accelerated aging at 
conditions of approximately 30ppm daily average monochloramines in water, and conversely chlorine in water, maintained at $45^{\circ} \mathrm{C}$ for 30 days in heated water baths. On the appropriate testing days samples were removed and cross sectioned for testing. On the testing days samples were also tested for changes in hardness and volume. Since data were needed concerning the effects of the disinfectants and water on the carbon black from the interior of the sample the specimens could not be reused on subsequent testing days and new coupons had to be cross sectioned. Samples were held in relative stasis by placement in distilled water at ambient temperatures, until testing could be accomplished. In addition to AFM imaging, Scanning Electron Microscopy allowed for high resolution images of unsaturated samples to be made, which helped to confirm the quantity and size of the carbon black particles. A modified form of Fick's second law was employed to derive an estimated diffusion coefficient of liquid diffusion into the polymer matrix for the three types of rubber tested in both water containing monochloramines and in water containing chlorine. In every case the effect of degradation on the rubber pieces by monochloramines was greater than the degradation induced from chlorine. 


\section{METHODS AND MATERIALS}

\section{A. Accelerated Aging and Determination of Effects on Bulk Properties}

Degradation of rubber used in the municipal water supply infrastructure occurs over a span of tens to even a hundred or more years. The effects are considered abnormally premature if the rubber parts need replacement in only a few years or months instead of within tens of years. In order to replicate the effects of long term degradation over a reasonable amount of time in a laboratory setting, methods of accelerated aging are employed. Aging in the municipal water systems occurs at ambient temperatures and with very low concentrations of disinfectant. This study focuses mainly on the use of monochloramines as the disinfectant, though effects of chlorine were also examined for comparison.

In order to accelerate the effects of aging, in the lab greater than ambient temperatures and increased concentrations of disinfectant are used. Research conducted by investigators such as Riber (1993) and Valleru (2006), attest to the fact that temperature increases cause accelerated elastomer degradation. Recently some researchers have also employed methods of accelerated aging by using higher than 1 to 
$5 p p m$ concentrations of the disinfectants as is standard for the municipal water supply. Similar methods of accelerated aging and subsequent determination of bulk properties has been conducted by scientists for the AWWA Research Foundation, which has also provided support for this study. It was determined that the optimal conditions for this study are at a temperature of $45{ }^{\circ} \mathrm{C}$, and an average concentration of disinfectant of 30ppm. This temperature was chosen to provide enough thermal energy to cause effects of aging at an accelerated pace without causing elastomer deterioration due to thermal degradation that is not common to aging. The optimal concentration was chosen to be similar to the concentration levels used in past research, allowing for comparisons to be made more easily. In addition higher concentration levels did not appear to have a substantially greater impact on the rubber samples.

Molded rubber samples of Natural Rubber, SBR and EPDM sulfur cured were obtained from the Ashtabula Rubber Company in the form of 6" x 7" x 0.08 " slabs. The rubber provided by Ashtabula was as prescribed by formulations used in the report by Riber (1993), as shown in Figures 2.1 to 2.3 formulas are given in units of parts per hundred of rubber, PHR. Some substitutions were made by the manufacturer to the formulations due to availability of materials. One of the substitutions was the use of carbon black SRF N762 in all three of the rubber formulations. Formulations for the rubber types used in this experiment were chosen for the base materials without the addition of substances which enhance resiliency to attack by ozone and disinfectants such as chlorine and chloramines. Results representative of all elastomers of the three types chosen were desired since some proprietary formulations may contain ingredients which 
impart special resistances only the base formulations were used. Test coupons with dimensions of 4" x 1" x 0.08 " were cut from the slabs using a die and press. The coupons were loaded into cylindrical stainless steel containers and were held apart for exposure on all sides to the water with disinfectant by positioning the coupons onto glass stands with four arms on top and four arms on bottom extended outward from a central vertical rod, visible in Figure 2.4. This configuration allowed 20 coupons to be placed in a vessel, each of the rubber types tested were placed in separate containers. Also visible in Figure 2.4 are the die and press used for cutting samples from the rubber slabs and a fully loaded stainless steel cylinder in which the rubber samples were emerged in test solutions.

\section{Table 2.1}

\section{Natural Rubber Formulation}

\begin{tabular}{|lll|}
\hline Ingredient & PHR & Function \\
SMR CV 60 & 100 & Standard Malaysia Rubber (latex) \\
SRF N762 & 70 & Carbon black \\
Sun 42400 (or equiv.) & 2 & Extending oil \\
Sulphur & 2.5 & Primary vulcanizing agent \\
ZNO & 5 & Curing system additive (activator) \\
Santocure & 0.7 & Accelerant - thiazole based \\
\hline
\end{tabular}


Table 2.2

Styrene Butadiene Rubber Formulation

\begin{tabular}{|lll|}
\hline Ingredient & PHR & Function \\
SBR 1502 & 100 & Styrene Butadiene Rubber base polymer \\
SRF N762 & 90 & Carbon black \\
Sun 4240 & 10 & Extending Oil \\
Sulphur & 1 & Primary vulcanizing agent \\
ZNO & 5 & Curing system additive (activator) \\
Stearic Acid & 1 & Curing system additive \\
Santocure & 1 & Accelerant \\
\hline
\end{tabular}

Table 2.3

\section{EPDM-S Rubber Formulation}

\begin{tabular}{|lll|}
\hline Ingredient & PHR & Function \\
Nordel 1070 & 100 & Ethylene-propylene diene copolymer \\
SRF N762 & 100 & Carbon black \\
Sun 22800 (or equiv.) & 110 & Extending oil \\
Sulphur & 2 & Primary vulcanizing agent \\
ZNO & 5 & Curing system additive (activator) \\
Stearic Acid & 2 & Curing system additive \\
Altax/Butyl Z/M Tuads & 4.8 & Accelerants \\
\hline
\end{tabular}



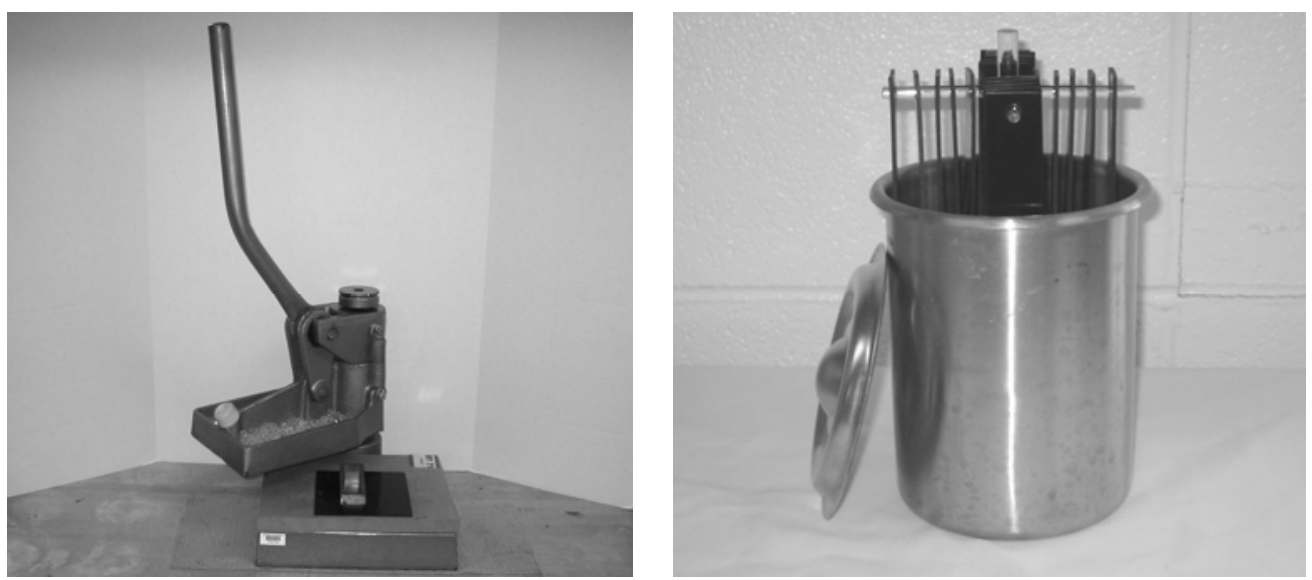

Figure 2.4 Rubber slab, die and press (left), samples cut and loaded in stainless steel cylinder (right)

Fresh solutions of disinfected water were made daily during a 30 day accelerated aging test period. Distilled water was used in preparation of the disinfectant based water mixtures. To this system $\mathrm{pH} 7.0$ phosphate buffer was added for stabilization of $\mathrm{pH}$ in an amount of $4 \mathrm{ml}$ per liter. Chlorine was added to the system in the form of sodium hypochlorite, when the presence of monochloramines was desired ammonium hydroxide was then added in a weight ratio of 2 to 1 , chlorine to ammonium. Additionally sodium hydroxide and a $\mathrm{pH} 7.0$ phosphate buffer were used to adjust the initial daily $\mathrm{pH}$ to between 8.2 and 8.4. Sodium hypochlorite was added in an amount as needed to achieve a concentration of about 50ppm of total chlorine which typically required about $1 \mathrm{ml}$ per liter of water. Ammonium hydroxide was added for the formation of monochloramines in an amount of about $1.6 \mathrm{ml}$ per liter. Solutions were changed daily with ending concentrations and $\mathrm{pH}$ being monitored. Initial daily concentrations were set for about 50ppm of chlorine or monochloramines in order to average about 30ppm. Concentrations were determined with amperometric titration utilizing phenylarsine oxide as the titrant, 
potassium iodide as an indicator and $\mathrm{pH} 4.0$ buffer for stabilization of the ions. A standard digital $\mathrm{pH}$ meter was used to determine the $\mathrm{pH}$ of the solutions. The glass fixtures loaded with rubber coupons were immersed into the stainless steel vessels containing the freshly made solutions. The covered stainless steel vessels were themselves immersed in circulatory water baths and maintained at $45{ }^{\circ} \mathrm{C}$. The initial ratio of chlorine to ammonium has a significant effect on the form of the aqueous chlorine produced and the amount of total residual chlorine formed as described by Snoeyink and Jenkins (1982). At the ratio chosen for this experiment a significant amount of residual $\mathrm{NH}_{3}$ is formed, but is in accordance with ASTM protocols. The products of the reaction vary with $\mathrm{pH}$ and contact time as well. By setting the initial $\mathrm{pH}$ at 8.3, monochloramines, $\mathrm{NH}_{2} \mathrm{Cl}$, are the predominant species formed (Schreck, 2006). Monochloramines were the preferred species over the formation of dichloramines, $\mathrm{NHCl}_{2}$, or trichloramines, $\mathrm{NCl}_{3}$. Slight daily adjustments were made to the formulation of the disinfectant solutions to achieve the desired concentration and $\mathrm{pH}$. Solutions were changed every day during the 30 day test periods. Samples were removed from the solution for testing on days 10, 20 and 30. Testing of elastomeric properties was accomplished as well as processing for analysis by AFM imaging. The water bath used to maintain testing temperature, the ampomeric titrimeter used for testing concentrations and the $\mathrm{pH}$ meter used are shown in Figure 2.5. 


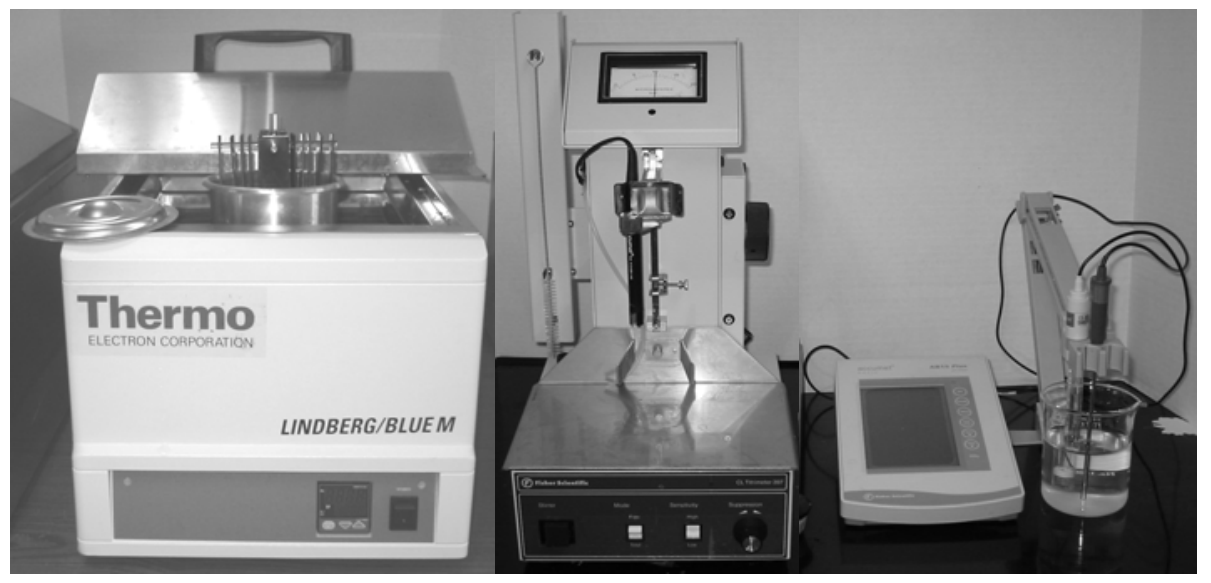

Figure 2.5 Water bath (left), titrimeter (center) and pH meter (right)

In addition to imaging of the samples during the accelerated aging cycle, tests were also made of changes in the elastomeric properties of the bulk material. All testing was performed on the sample coupons on days 10, 20 and 30 of the testing cycle as well as on day 0 , prior to accelerated aging. The elastomeric properties tested to determine changes in the bulk properties were changes in mass, volume and hardness. Three coupons of each type of rubber designated for elastomeric testing were removed from their solutions on the day of testing. The rectangular coupons were first weighed on an analytical balance while completely submerged in water. Changes in mass were determined by weighing the coupons on the analytical balance after being dried of superficial moisture. The submerged weights and dry weights were used to calculate volumes and their changes over the testing period. Changes in Shore A hardness values were determined using a digital durometer. The results from the three samples of each type of rubber were averaged in determination of mass and volume changes. Each of the three individual samples was tested in three different locations and averaged together for determination of changes in Shore A hardness. 


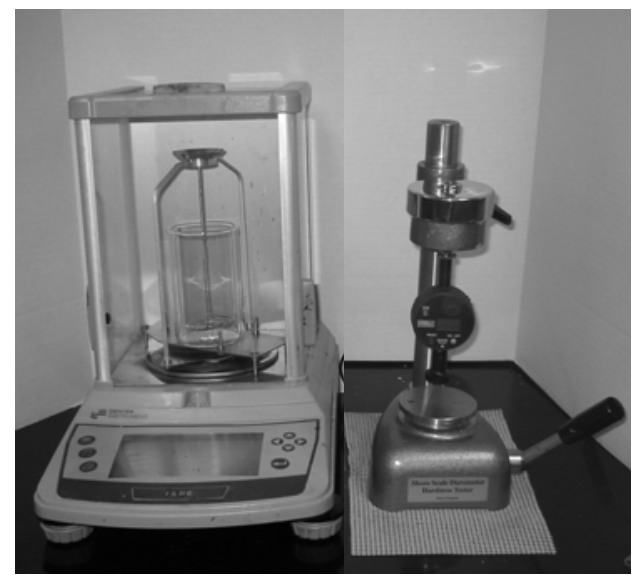

\section{Figure 2.6 Analytical balance with water submersion aperture (left) and digital durometer (right)}

\section{B. Cross Sectioning and Imaging with Atomic Force Microscopy}

Various methods for cross sectioning were analyzed. It was determined that any method in which the sample was cut using a blade would leave striations across the freshly cut surface that would make imaging on the nanoscale more difficult. Cutting the samples was also determined to be too invasive as artificial tearing, or removal, of the carbon black particles from the sample surface would have been a concern. As an alternative to slicing the sample coupons it was decided to try using liquid nitrogen to quickly freeze the coupon then breaking it by hand over a sharp edge such as a glass tile. Throughout the testing slight changes were made in this procedure. Another effective means of breaking the samples was to clamp half the sample in between two strips of metal, frozen using liquid nitrogen and then broken by hand. In some cases the sample simply shattered while freezing. In some cases the test coupons were slightly etched on one side before freezing. This aided greatly in obtaining a smooth break but made 
imaging of the sample difficult at the near edge location on the etched side, in some cases. On testing days, the samples designated for cross sectioning were removed from the disinfectant and water mixture. Pieces of cross sectioned material used for AFM analysis were taken from as close to the center of the sample as possible. This was to insure diffusion of the carbon black particles out of the sample and disinfectants into the sample occurred in only one directional axis across the smallest width of the sample, 0.08 inch. It was desired to develop a graphical representation of the effects on carbon black as the liquid penetrated into the sample over time so relatively thin samples in contrast to the other two dimensions were selected for the experiment. The coupons being only 0.08 inch thick compared to one inch wide and four inches in length were appropriate for the diffusion distance out through the larger dimension to be considered infinite. Diffusion effects were occurring across both faces of the sample but it is the supposition of the experimenters that diffusion does not fully reach the middle of the sample in the 30 day test period. This supposition is probably not completely correct but is supported to some extent by data analyzed in the following chapters of this report. 


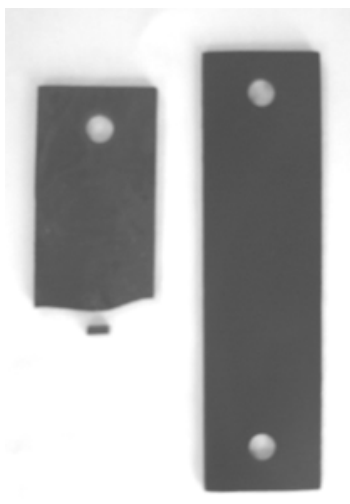

\section{Figure 2.7 Full sized rubber sample next to sample broken using liquid nitrogen and cross sectioned sample cut from near center appropriate for AFM imaging}

Cross sectioned samples without accelerated aging, day 0 samples, were imaged using Scanning Electron Microscopy. A continuous image from one side of the sample to the other across the 0.08 inch width was produced by placing consecutive images together. Additionally, higher magnification images were taken from the approximate center of each of the lower magnification images to more clearly show a consistent dispersal pattern of carbon black throughout the sample. After samples were removed from the disinfectant solution for cross sectioning they were kept in relative stasis by placing them in distilled water and holding them at room temperature for possible future testing and analysis. Samples used for AFM analysis were cut from as close to the center of the sample with respect to the one inch width and four inch length. The interior of the sample was then imaged from one surface to the other in 10 to 13 positions within a 20 micron square area respectively. The images taken were 4 microns square dimension and from one to four images were captured at each position. The positions extending from

one side of the sample to the opposite side were spaced apart by hundreds of microns, 
such that even though approximately 30 of the four micron square images were taken across the surface only a small fraction of the surface was imaged. In order to improve the representative value of the scans the data became grouped together even further by folding the sample data over on itself at the center of the sample. In this way only five to six positions existed from the center of the sample to the edge by grouping together areas that lie symmetrically distant from the center.

AFM imaging was accomplished on a Digital Instruments 5081JV Atomic Force Microscope with a Nanoscope IIIa Scanning Probe Microscope Controller made by Veeco Metrology Group (Santa Barbara, CA). After cross sectioning of the sample a small piece about 0.25 inches long, 0.08 inches wide and 0.1 inches thick from the center of the cross section was mounted onto a sample disk. The disk with sample was placed on the magnetic sample holder for the AFM. Upon turning on the AFM, a cantilever with tip mounted on a sub millimeter chip must be inserted and tuned. Silicon BS-TAP300 cantilevers (Ted Pella Inc., Redding, CA.) which had a drive frequency of 200 to 400 $\mathrm{KHz}$, a nominal radius less than $10 \mathrm{~nm}$, and a spring constant of between 20 and $75 \mathrm{~N} / \mathrm{m}$ were used for tapping mode imaging of the samples. The drive amplitude was between 20 to $220 \mathrm{mV}$ and the drive phase was $20^{\circ}$ to $180^{\circ}$. A scan rate of $0.5 \mathrm{~Hz}$ was used, with 256 lines of resolution. Amplitude set point was usually adjusted to between 1.0 and 1.4 Volts. An integral gain of between 0.2 and 0.3 was used along with a proportional gain of 0.3 to 0.5 . Images of the sample were four square microns. Height images were set to a one micron scale on the $\mathrm{z}$ axis. Phase images were done on a scale of $100^{\circ}$. The images were adjusted for contrast, brightness and scales using the Nanoscope version 
6.13 software, by Digital Instruments. Many of the phase images were also flattened using the nanoscope software. In interest of manipulating the images as little as possible a zero degree flatten was generally used, in some cases a first or second degree was used. Images were usually flattened in both the $\mathrm{x}$ and $\mathrm{y}$ planes. The flattening is used to remove slopes and curvatures that may be added to an image due to issues with the scanner, thermal drift in the cantilever or some other instrumental condition. In the case of a 0-order flattening, the software is removing a voltage offset that is caused by the positioning of either the cantilever with respect to the surface or of the laser on the photodiode. Further adjustments were made to the phase images using ImageJ software and analyzed. The ImageJ software converted the image into a binary form of white background and roughly spherical shapes corresponding to the harder material in the expected size range and quantity of carbon black. Data from ImageJ was gathered, area fractions were given directly, while effective diameters were calculated from average particle size which was also given directly by the software. The number of particles present was also given, as well as the total area covered by the particles. The data was recorded then graphed using Microsoft Excel.

Scanning Electron Microscopy was used to supplement data from the AFM. Samples that had not yet been aged and were therefore not saturated with water could be imaged using the SEM. Saturation of the samples during accelerated aging prevented the use of the SEM due to gasses expanding upon exposure to the electron beam. The SEM uses electrons to form a beam produced at the top of the microscope by heating of a metallic filament. The electron beam follows a vertical path through the column of the 
microscope passing through electromagnetic lenses which focus and direct the beam. Once the electron beam hits the sample other electrons called backscattered or secondary electrons are ejected from the sample. Detectors collect the secondary or backscattered electrons and convert them into a signal that is sent to a viewing monitor. The use of the electron beam allows for images with a resolution of 5 nanometers. This resolution makes more precise definition possible than images obtained using AFM tips with a nominal radius of approximately 10 nanometers. Since the SEM uses electrons to produce images, most SEM's require that the samples be electrically conductive. Environmental SEM's are now available which can be used to view non-conductive or possibly wet samples. All metals are conductive; however non-metals such as elastomers must be covered by a thin layer of a conductive material such as gold. This was accomplished using a sputter coater which uses argon gas and a small electric field to blast atoms from a gold foil which settle onto the surface of the sample. The day 0 images produced by the SEM helped to confirm even distribution and size ranges of the carbon black particles in the polymer matrix.

Digital calipers and an optical microscope were used to determine the saturation depth of the disinfectant water mixture into the rubber samples. The liquid nitrogen used to freeze the sample coupons before breaking helped to bring out the appearance around the perimeter of the sample of the bulbous saturated region, visually obvious, with a flat inner core. Samples that had been sliced with a blade typically caused a cupped edge which dissipated the visibility of the bulbous region. This zone of saturation or expansion around the perimeter of the sample became thicker over time during the 30 day 
test periods. In order to determine an approximate rate of diffusion of liquid into the sample over time, this zone of expansion was taken to be indicative of the depth of penetration of liquid into the sample. Digital calipers were used to measure the change in width of the expanded area around the perimeter by subtraction of the unaffected inner core. This data was used in a modified form of Fick's second law to determine estimated diffusion coefficients in the two disinfectant solutions for the three types of rubber chosen for the experiment. 


\section{RESULTS AND DISCUSSION OF RESULTS}

\section{A. Introduction}

In order to produce images of the carbon black particles and evaluate their degradation during accelerated aging, the various modes of Atomic Force Microscopy were investigated and evaluated for effectiveness. In tapping mode, phase imaging allows for two dimensional phase images to be produced based on variations of hardness across the sample surface. This takes advantage of the distinct differences in the physical properties of the hard carbon black as compared to the soft polymer matrix into which it is embedded. Tapping mode accomplishes this by allowing some contact to be made with the surface and was thus determined to be the preferred approach in this experiment in order to take advantage of the sharp contrast in hardness between the carbon black and the surrounding matrix. Phase images produced in true non-contact microscopy are based on chemical properties, rather than physical. Carbon is the main constituent in both the carbon black and in the polymer chains thereby making phase imaging based on chemical properties, such as in non-contact mode, more difficult. For the purposes of this experiment the phase imaging has been done in tapping mode due to the need to sense the physical property change. Images obtained through Atomic Force Microscopy have been 
adjusted for contrast, brightness, and scales of height and phase. Images and data were evaluated using the analysis tools in the Nanoscope software and ImageJ analysis software. ImageJ is a freeware analysis tool, which converts the image data into a binary form, then evaluates the image for particle size and area fraction covered by the particles.

The elastomers used in this study contained Semi Reinforcing Furnace grade (SRF) carbon black. Samples of the carbon black powder obtained from the manufacturer have been determined to fall into the expected size range of 60 to $100 \mathrm{~nm}$, using scanning electron microscopy (SEM) shown in Figure 3.1. It is evident that carbon blacks rarely appear as single spherical particles, but usually as aggregates of coalesced, fused elementary particles. Aggregates have a tendency to agglomerate into larger units, which may be redispersed by shear forces, but reagglomerate on standing. (Donnet et al., 1976)

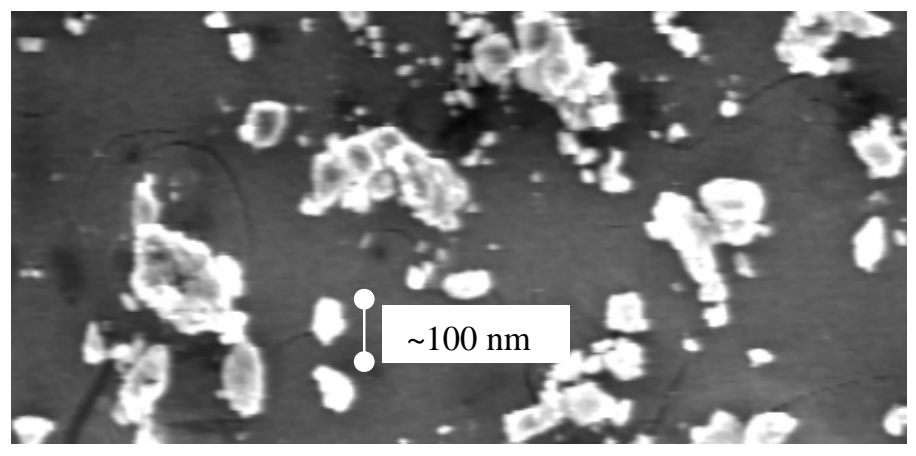

Figure 3.1 SEM image of carbon black powder (SRF) coated with gold 
Elastomer formulations obtained from the manufacturer were used to convert the theoretical volume fraction of carbon black into two dimensional area fractions. Evaluation of unused rubber samples indicated a reasonable agreement between the theoretical area fraction determined by formula estimation and the area fractions obtained using SEM, AFM and ImageJ. Natural Rubber was estimated to have an area fraction of about 25\% carbon black. SBR and EPDM sulfur cured rubbers were estimated to have an area fraction of about 30\%. Scanning electron micrographs of virgin samples of the three pertinent rubber types are shown in Figures 3.2, 3.3 and 3.4. The micrographs shown in the figure are of a cross sectioned 0.080 inch thick coupon. Cross sectioning was done using liquid nitrogen to freeze the coupon and subsequently breaking by application of force either by hand or with the aid of a flat surface with a sharp edge, additionally shattering would sometimes occur while freezing. Freezing and breaking was done in order to obtain a cross sectioned inner surface virtually free of mechanical abrasion as mentioned previously.

The cross sectioned samples imaged with a Scanning Electron Microscope in Figures 3.2 to 3.4 were coated with a thin layer of gold by chemical deposition to allow the electron beam to non-destructively image the sample surface. SEM technology uses an electron beam to scan the sample surface, thereby causing charging to occur which can damage softer surfaces such as polymers. Particles in the expected size range for carbon black appear to occur regularly over the sample surface. Consistent dispersal of the carbon black throughout the polymer matrix appears to be achieved during manufacture of the elastomer. The micrographs show that it is reasonable to assume that differences 
in particle size and distribution in aged samples is due mainly to the effect of the aging process, and not due to improper mixing during the elastomer manufacture or settling during curing. Moreover, analysis using ImageJ software shows that the area fraction of the two dimensional surface is in the expected range for each type of rubber and consistent with the Atomic Force Microscopy images. All subsequent scans of the rubber samples were performed using the AFM due to the release of expansive gasses from the rubber samples caused by the electron beam of the SEM that had become saturated with water during the aging process. The images in Figures 3.2 to 3.4 show the entire cross section from one side to the other for each type of rubber. At the magnification used to show the entire surface it is difficult to see the carbon black particles as they appear only as tiny white specks, so in addition, close-ups of specific regions located near the center of the band have been positioned directly below the main image. In the more highly magnified micrographs the carbon black particles can clearly be seen to be evenly distributed throughout the matrix. 

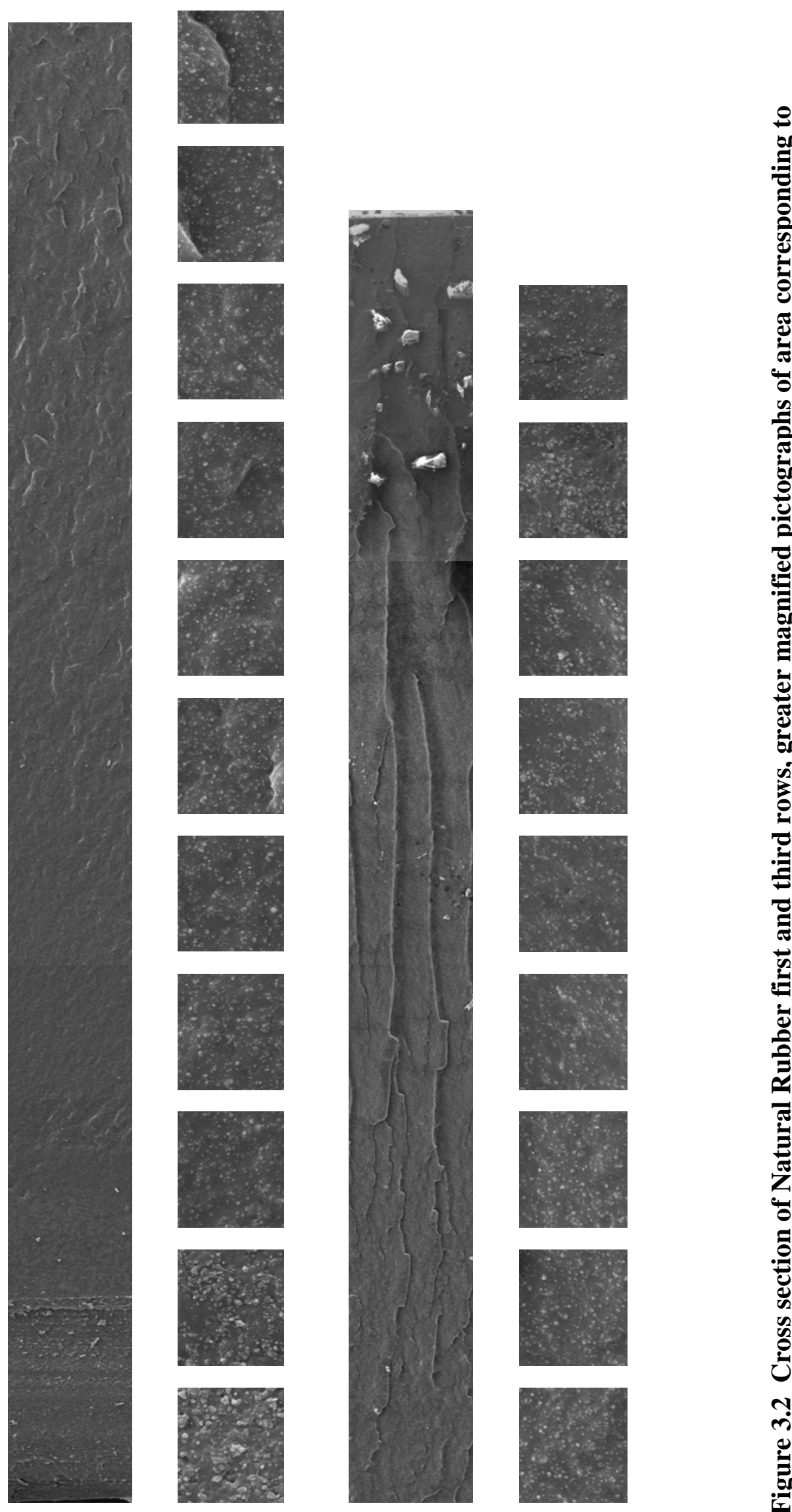


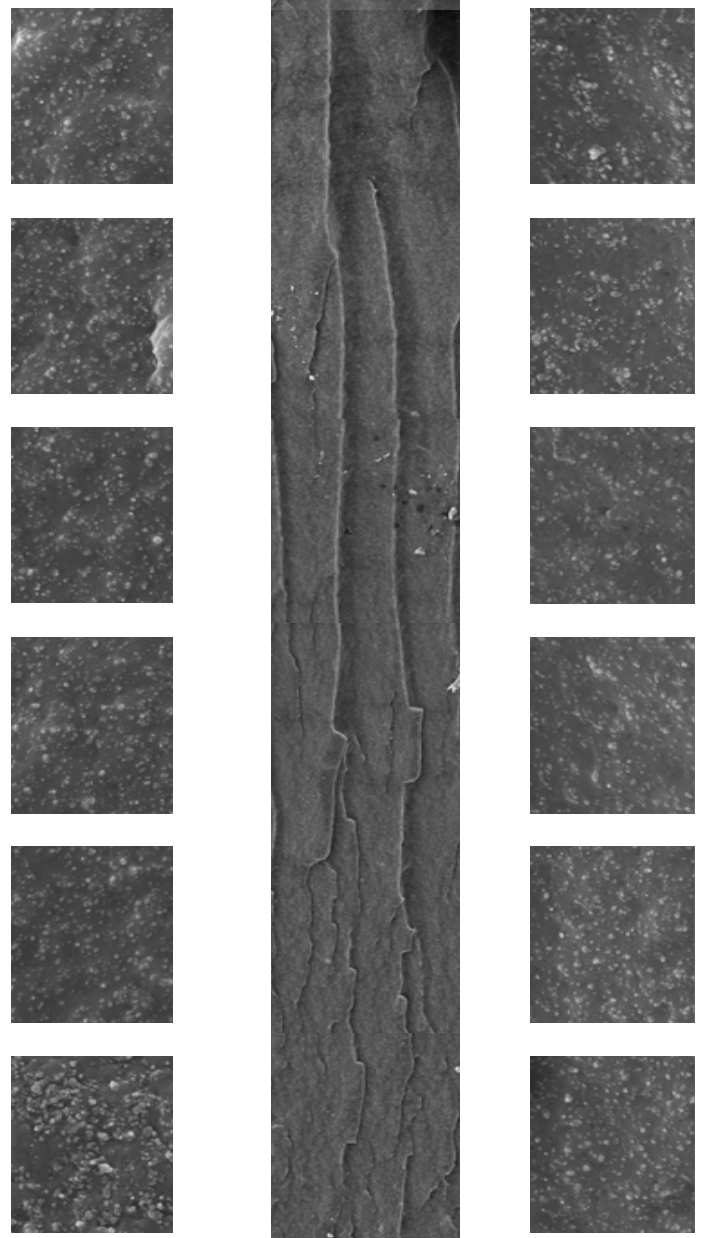

믈

ס્ّ.

है

를

胥

氖

क्ष

¿

है
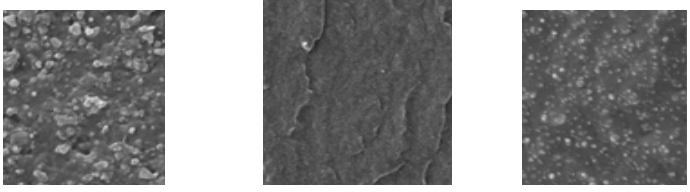

है है

艺

항

.

$\infty$ 응

岂 छే:

ก

望 

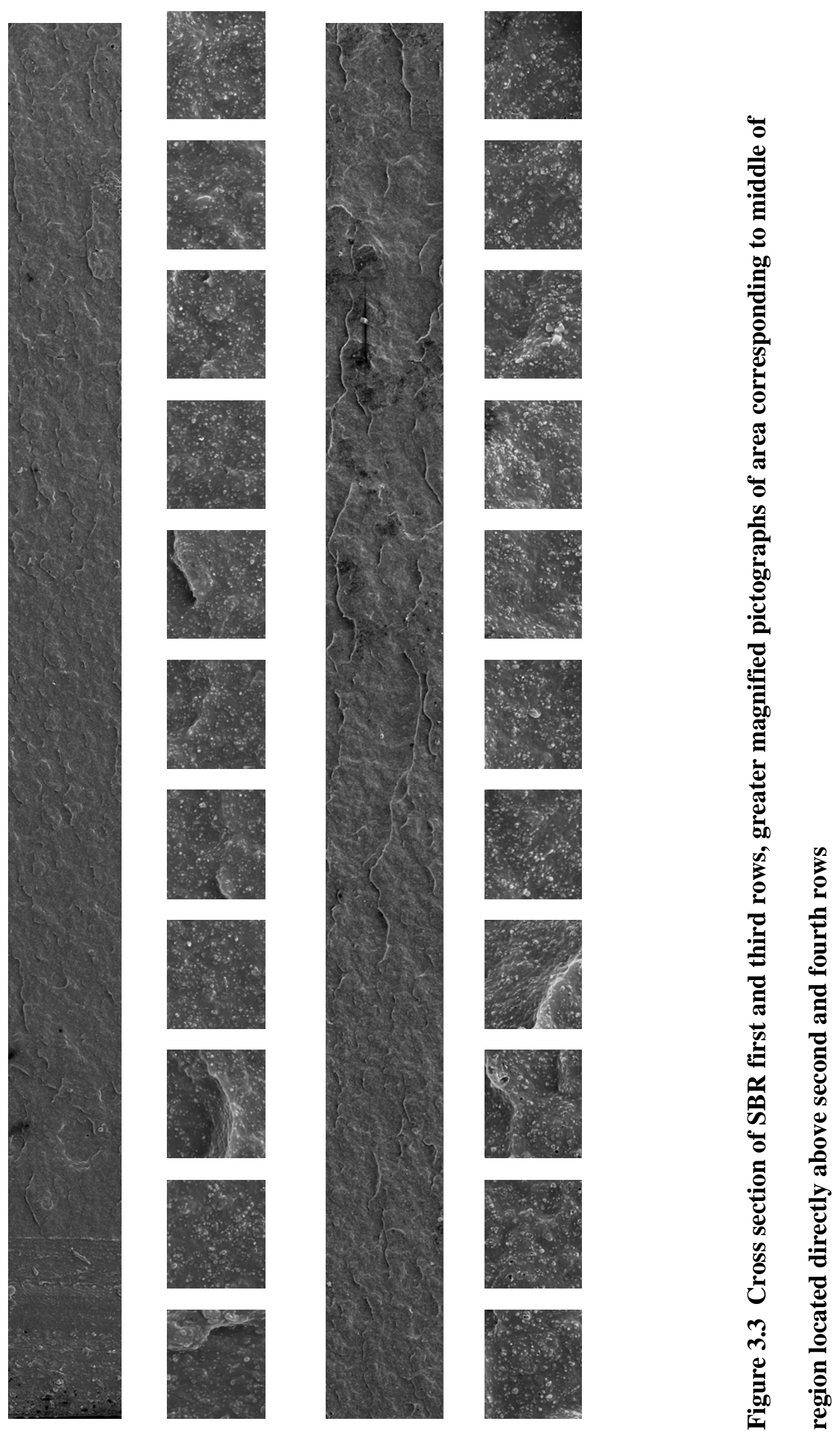


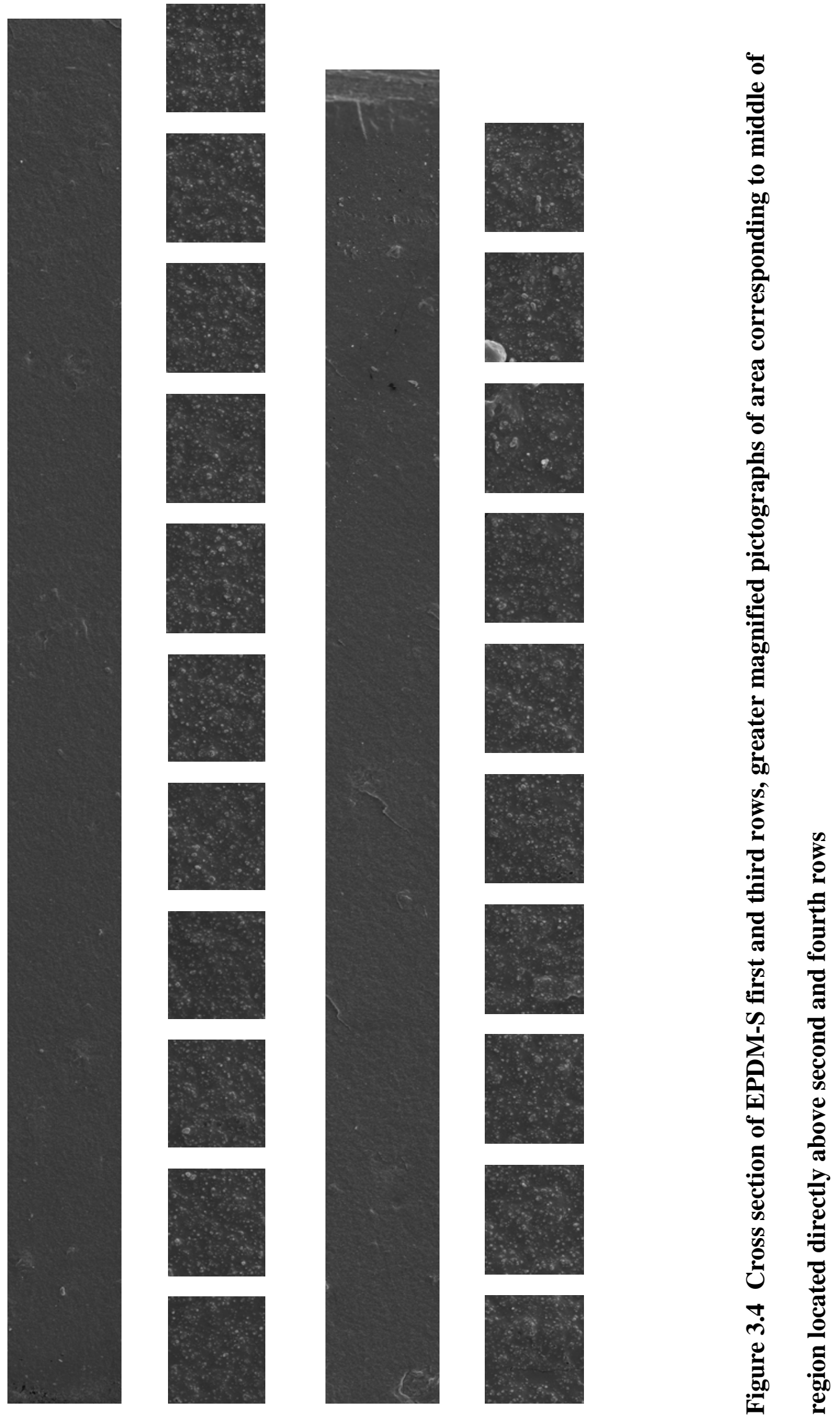




\section{B. Testing of Elastomeric Properties}

As in previous studies by such researchers as Reiber (1993) and Rockaway (2007), the effects of the system using monochloramines shows much greater degradation of the rubber than does the system using chlorine as the disinfectant. Testing for changes in hardness, mass and volume of the samples during the testing period were consistent with the effects seen in previous testing done at similar conditions. Figures 3.5 through 3.7 show the effects of accelerated aging on the elastomeric properties for each of the elastomers. Both disinfectant systems are shown for comparison. In each case the elastomeric properties of Natural Rubber and SBR show very similar results, while the degradation of the EPDM-S sample is substantially less. The similarities in elastomeric properties between Natural Rubber and SBR are probably due to the similar molecular arrangement of the backbone material that comprises their polymer chains. The most substantial difference is the existence of a small methyl group in Natural Rubber, or its synthetic form polyisoprene, as opposed to a large benzene ring structure that occurs in one out of every two or three repeat units in the polymer chain of SBR. 
Weight Change

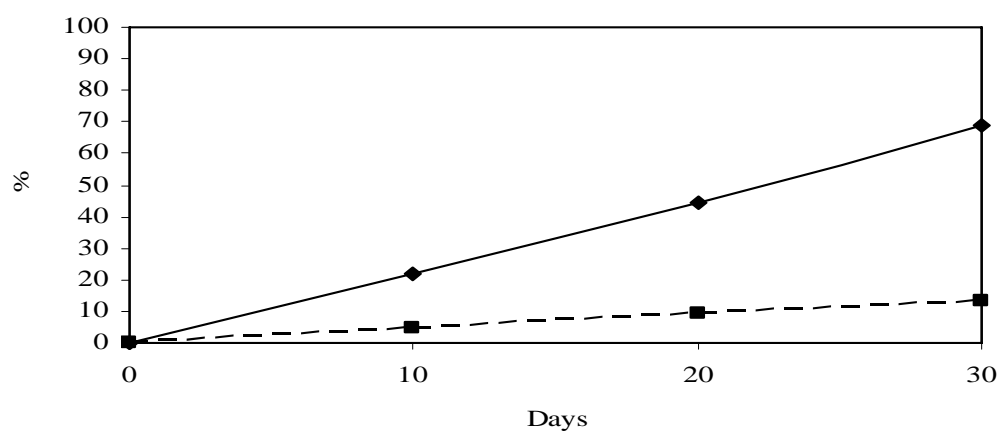

Volume Change

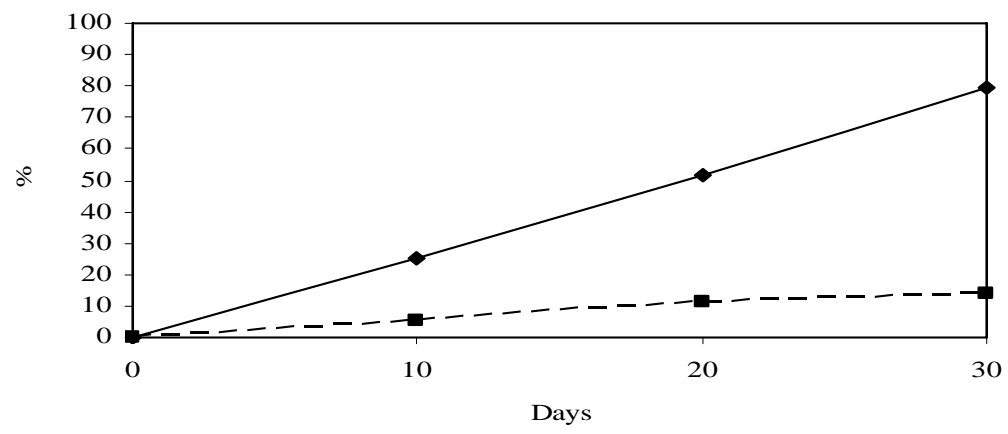

Hardness

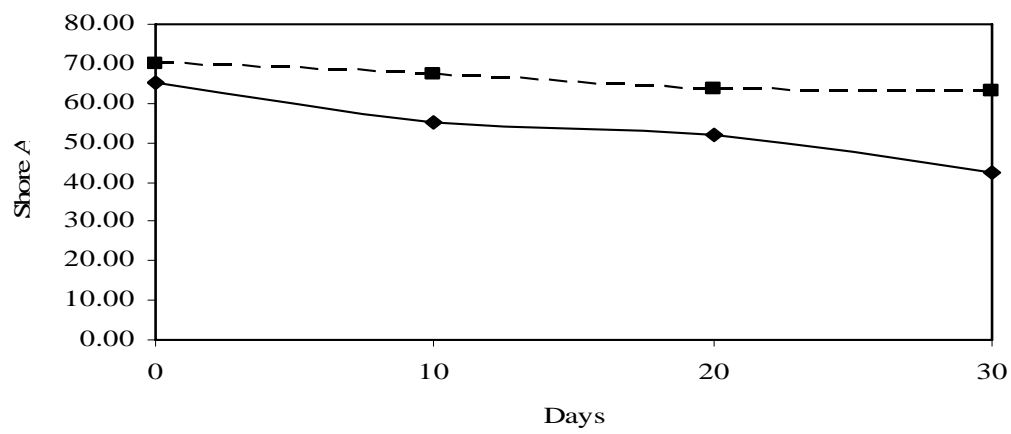

$\rightarrow$ Chloramines -- Chlorine

Figure 3.5 Effects of Monochloramines and Chlorine in water on elastomeric properties of Natural Rubber 
Weight Change

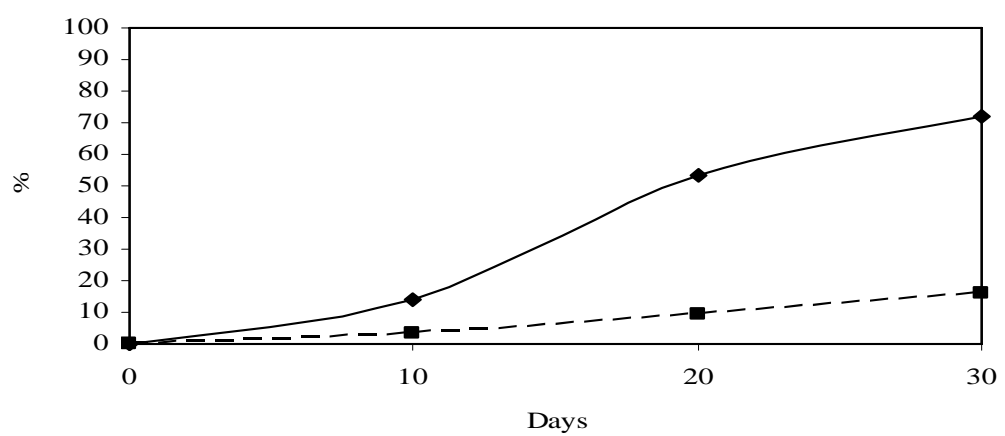

Volume Change

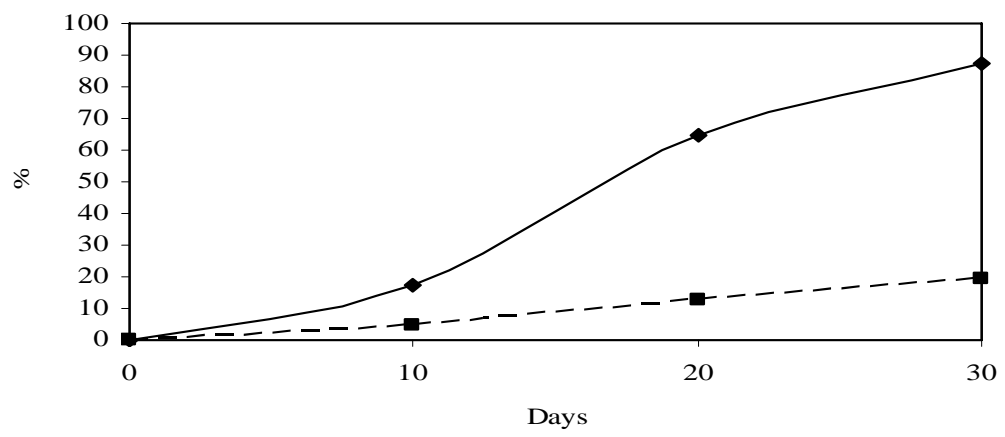

Hardness

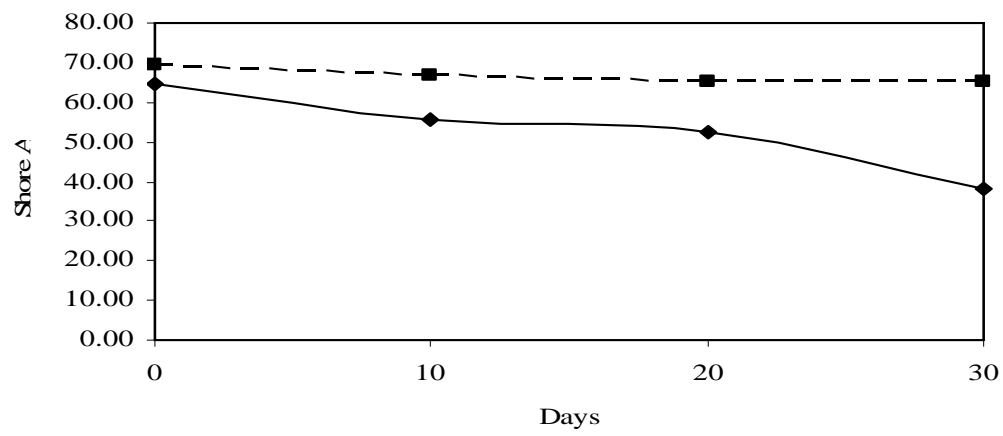

$\rightarrow$ Chloramines - Chlorine

Figure 3.6 Effects of Monochloramines and Chlorine in water on elastomeric properties of SBR 
Weight Change

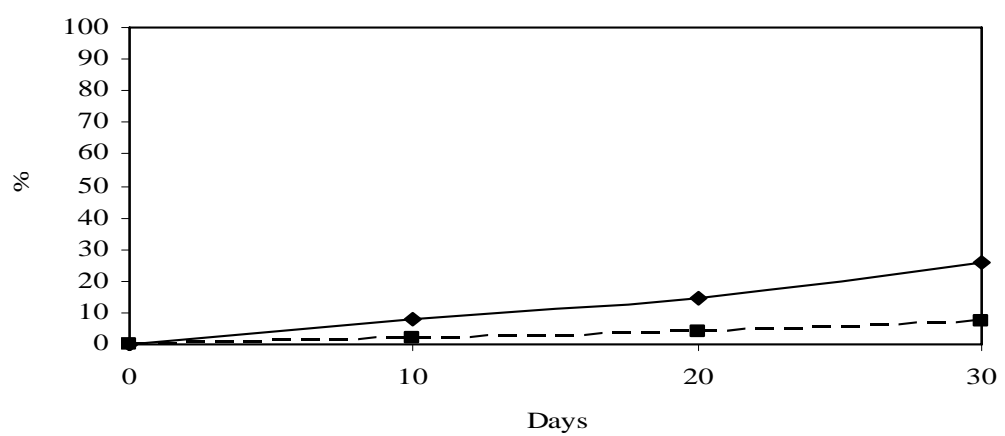

Volume Change

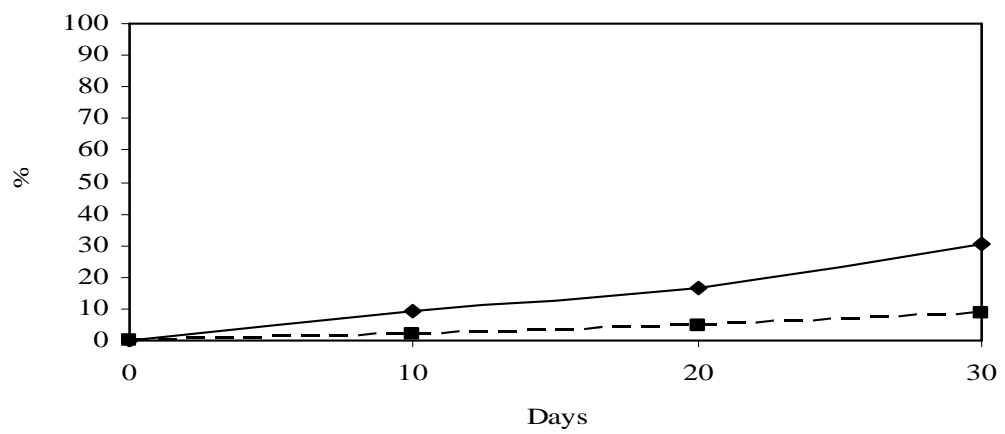

Hardness

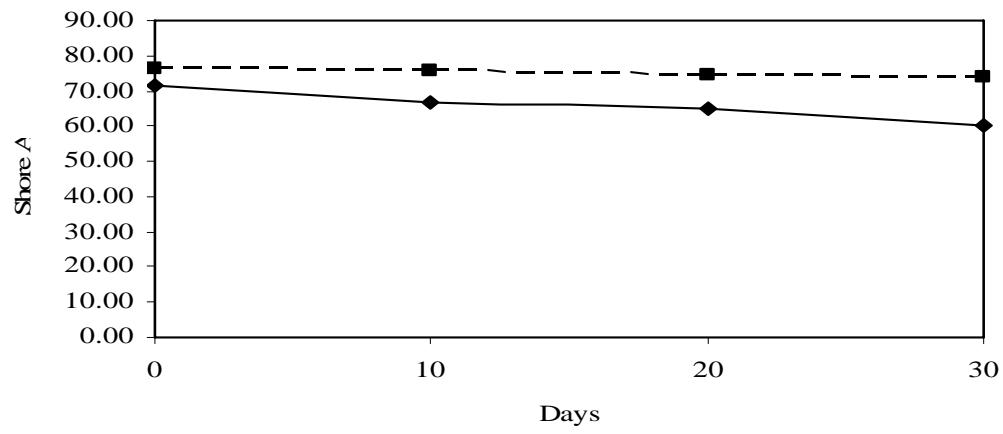

$\rightarrow$ Chloramines $\rightarrow-$ Chlorine

Figure 3.7 Effects of Monochloramines and Chlorine in water on elastomeric properties of EPDM-S 
Typically it has been shown that SBR performs slightly better with less deterioration of the elastomeric properties than does Natural Rubber. In addition to SBR performing slightly better under conditions of accelerated aging it has also been determined to have slightly lower diffusion coefficients of invasive fluids than Natural Rubber. The lower diffusion coefficients coincide with slower diffusion of liquid penetrating into the sample. The ability of SBR to maintain integrity somewhat better than Natural Rubber upon aging in aqueous solutions may be attributed to its slightly lower rate of diffusion and this in turn may be attributed to the large benzene ring structure that occurs regularly along the polymer backbone, effectively blocking diffusion of the invasive liquids and acting to block the removal of materials such as carbon black out of the polymer matrix. Figures 3.5 and 3.6 which show bulk property changes for Natural Rubber and SBR although very similar do display some slight differences. One such difference is the appearance of a step effect in the volume change of SBR as compared to a very straight curve of volume change in Natural Rubber. This may indicate that the effect of the benzene ring in SBR provides a temporary resistance to the penetrating fluids, which gives way about half way through the test cycle. Effects on bulk properties of EPDM-S as shown in Figure 3.7 are much less severe than the effects on Natural Rubber and SBR. Solutions using chlorine appear to have very little effect at all on the bulk properties of EPDM-S. The effects of solutions using monochloramines though substantially greater than effects from chlorine solutions are much milder than for Natural Rubber and SBR. 


\section{Atomic Fore Microscopy Data}

In this experiment, there was some difficulty in obtaining clear AFM images due to mechanical issues with the AFM as well as structural issues within the elastomer matrix. Atomic Force Microscopy can be a physically invasive process, as images are produced by a cantilever and tip moving across the sample surface. Touching the sample surface may allow for small particles of the elastomer to become attached to the cantilever tip. This could dramatically affect the images by increasing the effective radius of the tip, thus causing a decrease in the ability of the microscope to determine accurately the size of particles smaller than the effective diameter of the tip.

Variations of physical properties including phase differences also occur in the elastomer due to the presence of bound rubber. Carbon black interacts with the surrounding rubber rendering a portion of the rubber insoluble in normally good solvents. The bound rubber is harder than the soluble rubber matrix (Donnet et al., 1976).

Another source of uncertainty in the phase and thus the determination of size of the carbon black particles in the interior of the elastomer sample can be caused by the sensitivity of the AFM tip to slightly subsurface particles and subsurface edges of the protruding carbon black particle. The relatively small area covered by the AFM scans, only four square microns, also may allow for variations in particle displacement to become more evident than in the larger SEM scans. Inaccuracies in estimations of 
particle diameter seem to be especially acute where large agglomerations of particles exist.

Despite these difficulties a large number of AFM scans were made of the samples and have provided a substantial amount of pertinent data. The data concerning particle size and area fractions for those samples subjected to accelerated aging in solution containing monochloramines as the disinfectant have been analyzed using analysis tools such as the Nanoscope software and ImageJ as described above the resulting data was then graphed in time lapsed sequences.

AFM images of the 0.08 inch thick cross section were taken at regular intervals from one side of the sample to the other. Additionally data is grouped into approximate locations. Data represented as being at 0.02 inches is a composite of data from that approximate distance on each side of the midpoint. Etching of the sample on one side during breaking, and softening of the sample during aging, made obtaining useful scans difficult in many cases on one side of the sample but not as difficult on the other. For this reason the data is represented graphically from the center of the rubber coupon to the edge. The data gathered from across the entire width of the sample is effectively folded over on itself.

For the original, 0 day, samples, and for those sample for which very little change in volume had occurred the x-axis extends from 0 to 0.04 inches. The 0 point represents the middle of the sample and extends out to the edge of the sample. For samples where a 
significant amount of expansion had occurred the x-axis has been represented appropriately, such as in Natural Rubber and SBR at 30 days where the $\mathrm{x}$-axis is shown to extend out to 0.06 inches. Data points in the graphs as explained are a composite from that approximate location from both sides of the midpoint. Each point represents the average data gathered from at least two or more scans, in many cases four or more scans may make up the composite data. Error bars in the area fraction graphs have been represented indicating the range of data gathered from the approximate location. In graphs concerning average diameter of the particles, error has been calculated using variations from across the entire sample, due to limitation in the accuracy of phase imaging at the nanoscale level and difficulties in assessing particle dimensions. A vertical line denoting the original, 0 day, sample size has been added to graphs where the $\mathrm{x}$-axis has been extended.

Three dimensional surface images of the sample were made at the same time that phase images were produced. The purpose of rendering the 3-D surface plots is to help scrutinize the data produced by phase imaging. Since phase images are produced using differences in physical properties a void space left by a carbon black particle could be indiscernible from a protruding carbon black particle. The two may be represented in the same way, by the AFM, and both could appear as similarly colored spots in the expected size range on the computer depiction of the sample. In order to avoid this erroneous data, height images were analyzed, in conjunction with the phase images. Samples of typical phase and height images are shown in Figure 3.8. 

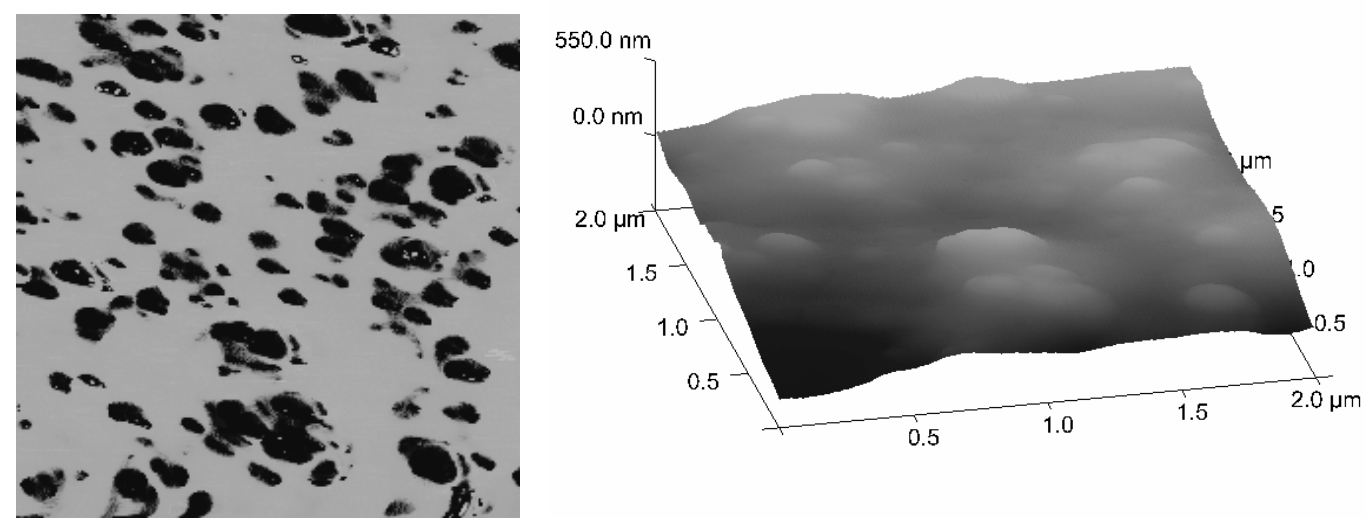

Figure 3.8 Phase image (left) and 3-D surface plot (right) of EPDM-S sample after 10 days accelerated aging in monochloramines mixed with water 

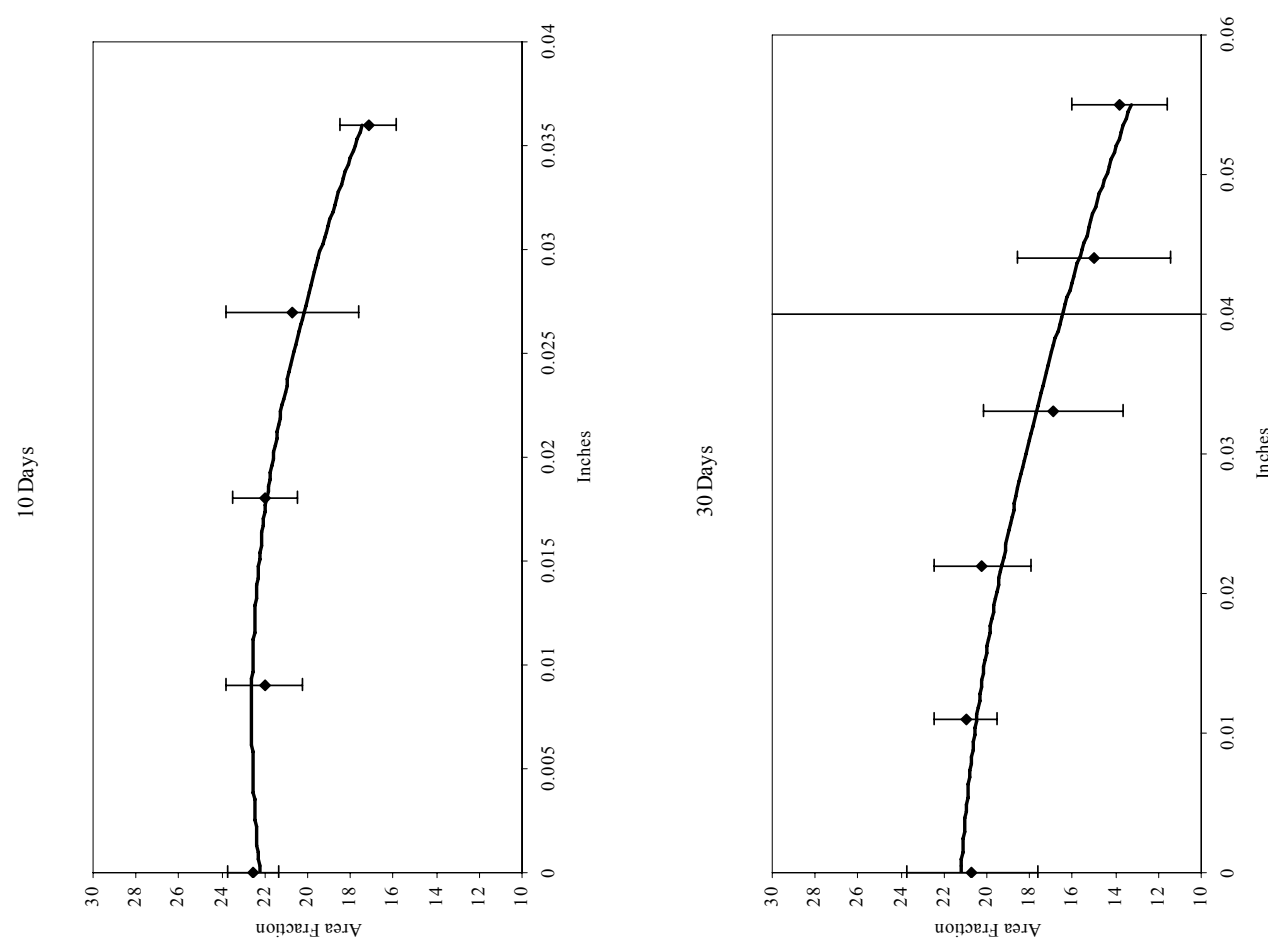

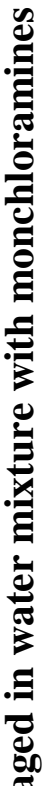
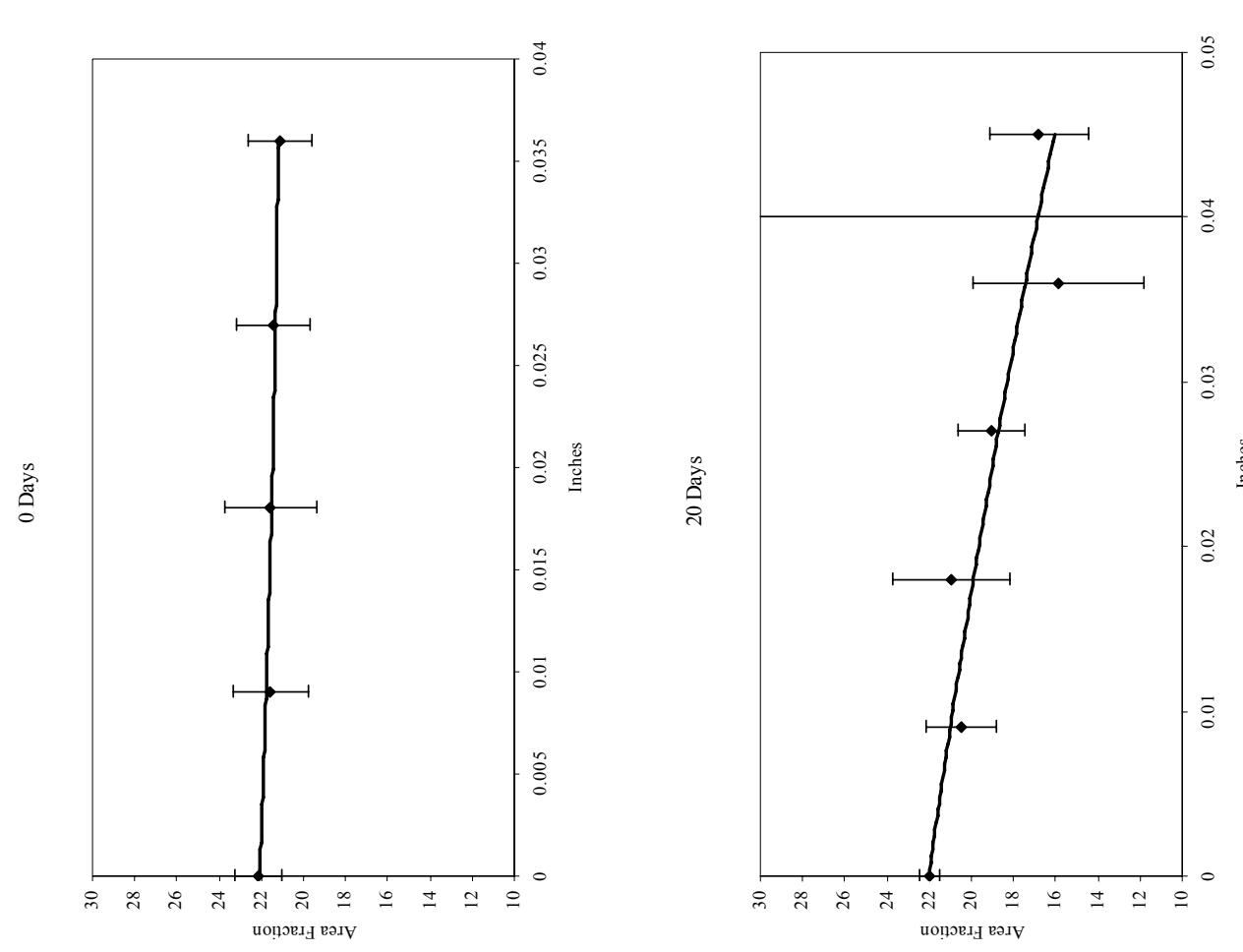

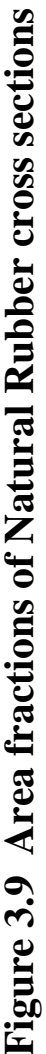



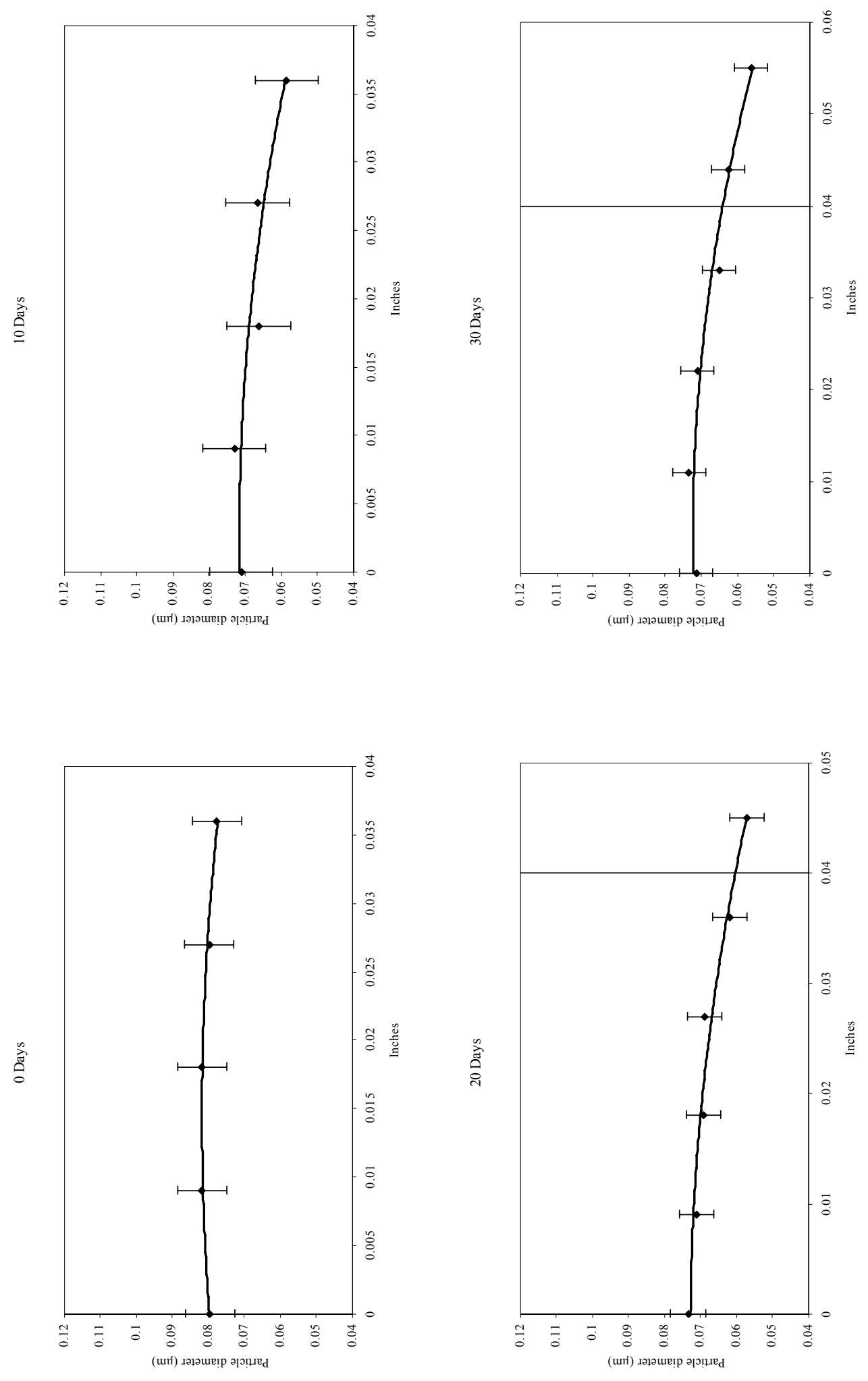

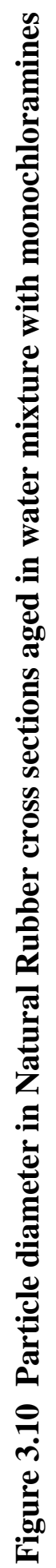


In Figures 3.9 and 3.10, representing the natural rubber samples, decreases in area fraction and particle diameter can clearly be seen. The graphs of area fraction covered by the carbon black show trends of severe initial degradation near the edge of the sample giving the 10 day graph a curved appearance. In the 20 and 30 day samples it appears that the effects of degradation become more consistent through the sample. The trendlines show an almost straight line indicating very little effect near the center and severe degradation near the edges. It can be reasoned that the area fraction would decrease at the outer edges of the sample due to expansion of the rubber as it becomes infiltrated with fluid, and cracks in the rubber are formed. It is more important to note the decrease in effective particle diameter. Analysis of AFM images using ImageJ software shows that the actual number of carbon black particles fluxuates slightly over time but does not decrease significantly in either disinfectant environment. If area fraction were the only parameter accounted for in the analysis of the AFM images then there would be very little evidence for the mechanism of loss of the carbon black. The greatest change accounting for the shrinking area fraction covered by the carbon black particles is the diminishing size of the particles. Complete loss of carbon black particles occurs mostly at the edge of the sample. This effect has to be tempered by the fact that the mass of rubber around the perimeter of the sample is expanding, and therefore smaller area fractions would be expected. Effects of degradation can be seen in Figure 3.11, depicting near edge and center of the sample conditions typical after 30 days accelerated aging. 

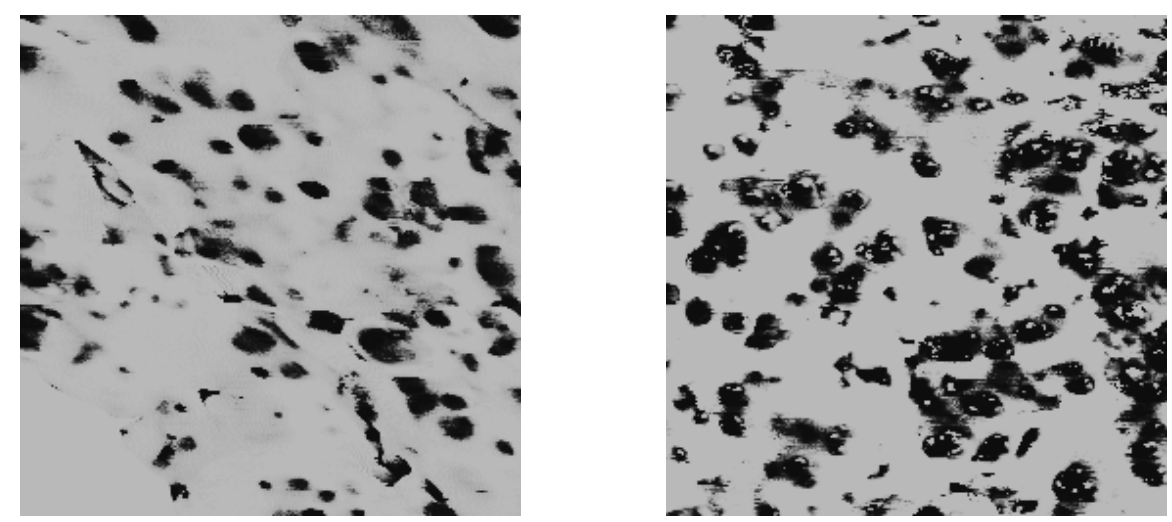

Figure 3.11 AFM images of Natural Rubber depicting effects of aging at the outer edge (left) and near the center (right) after 30 days aging with monochloramines 

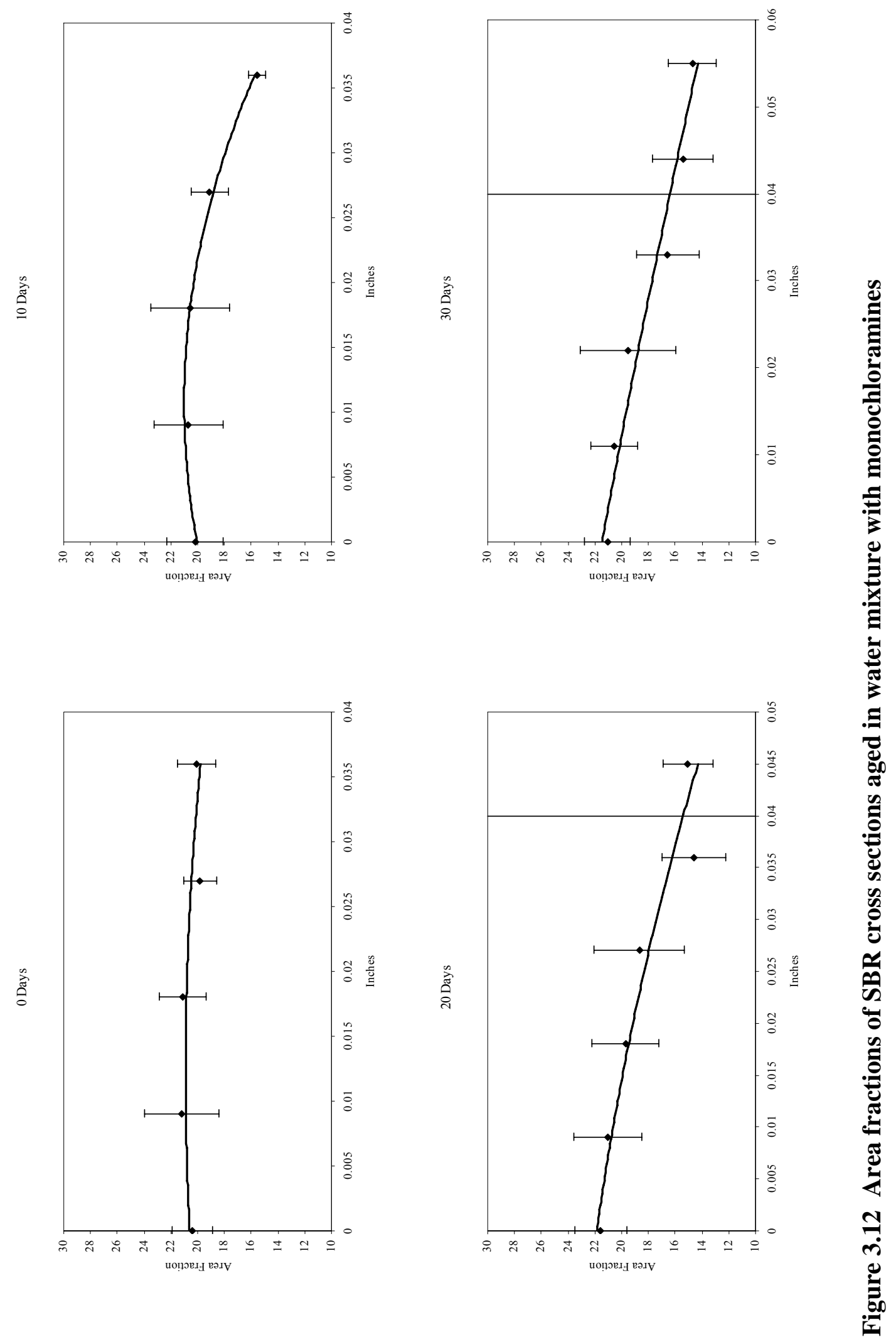

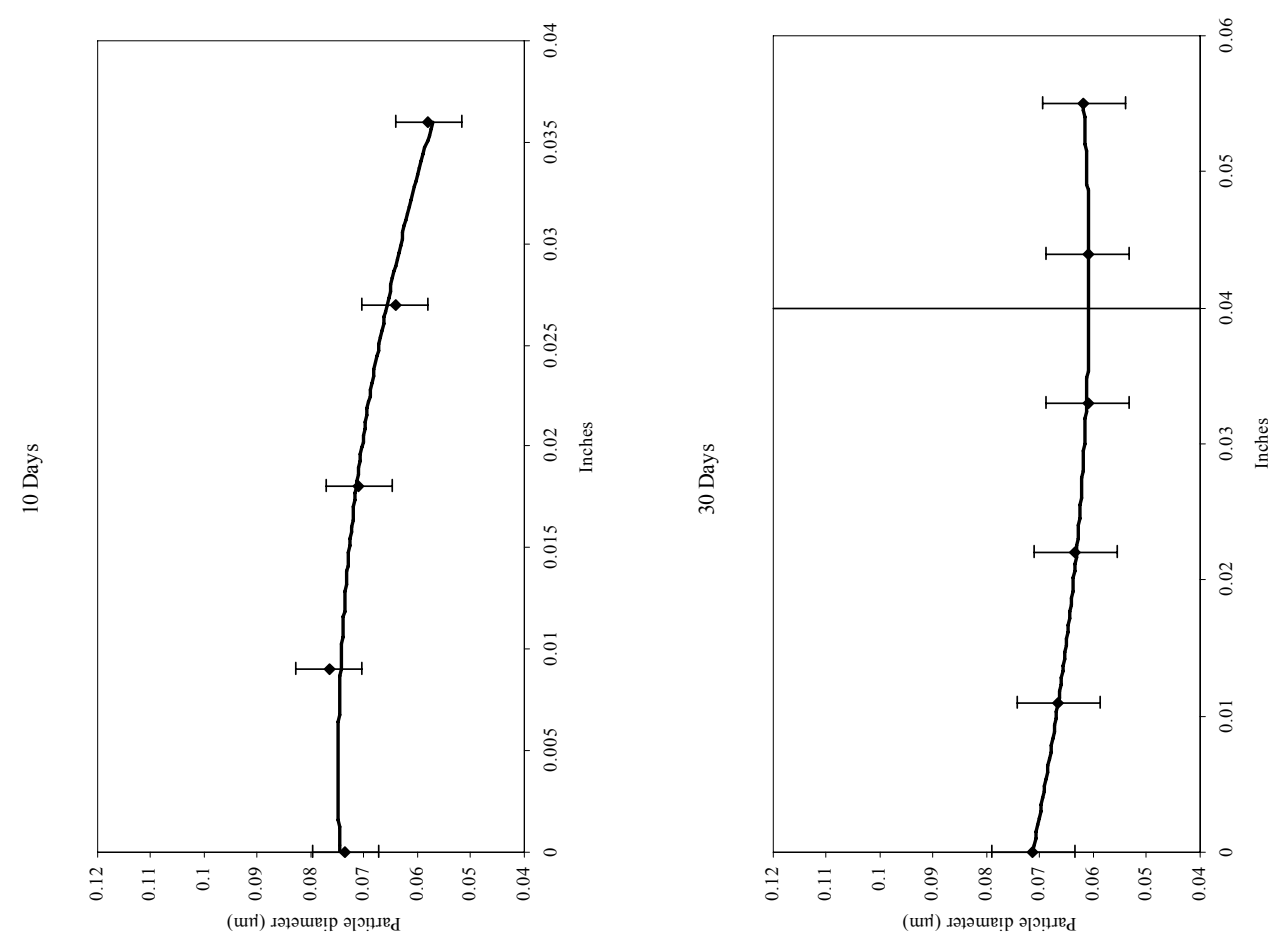

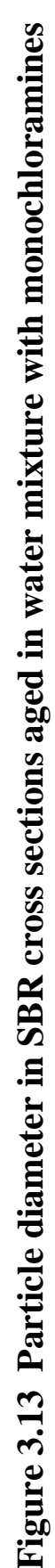
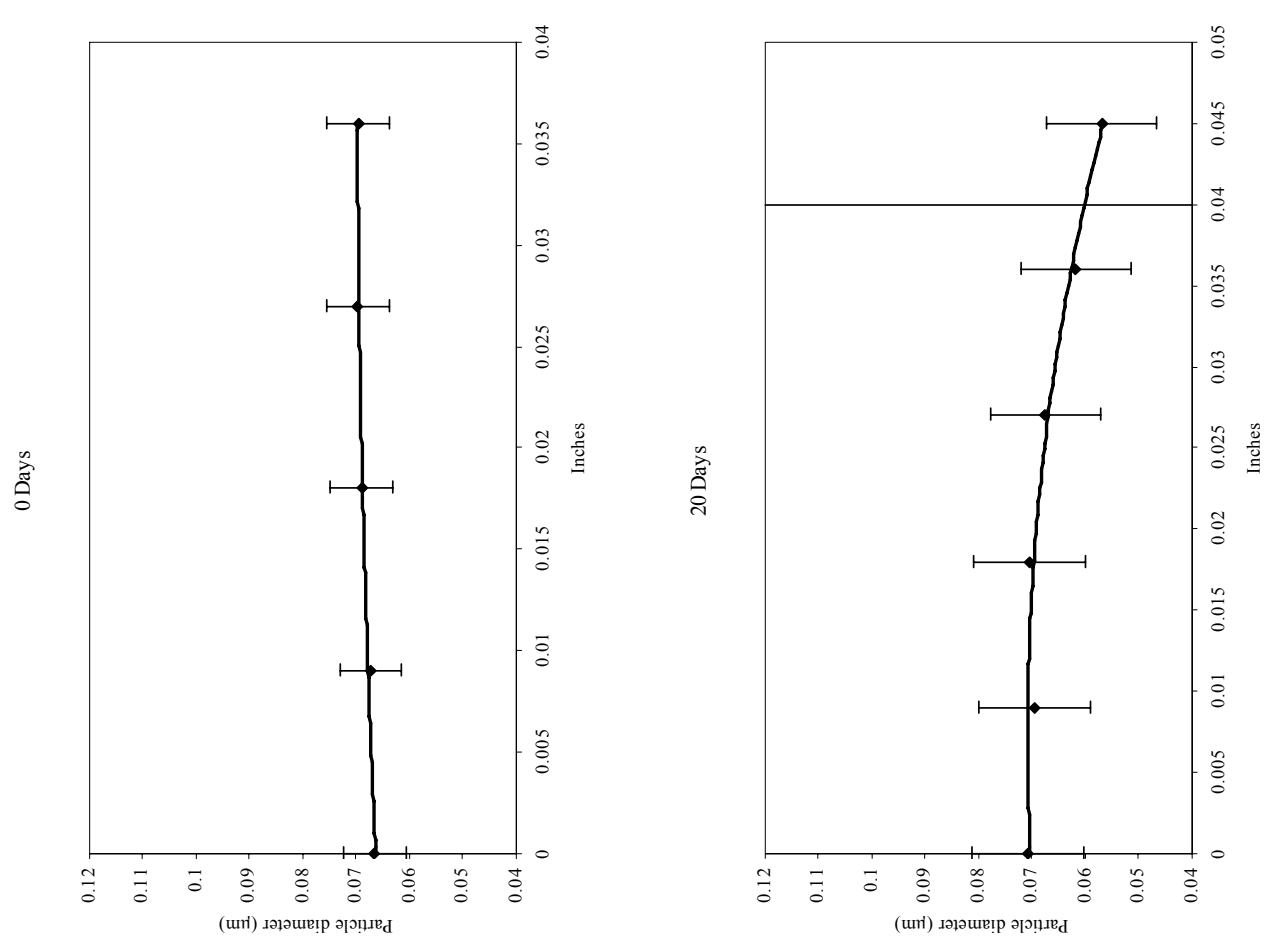
Similar trends to those of Natural Rubber can be seen in Figures 3.12 and 3.13, for SBR. Testing of the elastomeric properties of SBR during accelerated aging showed very similar trends of degradation to those of Natural Rubber so it is not surprising to see very similar trends in the loss of carbon black. Similarities in the composition of the back bone material in these two types of rubber account for their general similarities. The semi-crystalline structure of EPDM-S is probably most accountable for the longevity of life under adverse conditions (Osswald, 2003). In the cases of Natural Rubber and SBR which are pure elastomers no crystalline structure exists in the material. Natural Rubber and SBR also probably degrade more rapidly due to the location of the crosslinks compared to EPDM-S. Crosslinks in Natural Rubber and SBR occur between the polymer chains directly onto the backbone. EPDM type rubbers are terpolymers of ethylene, propylene and a small percentage of a nonconjugated diene providing unsaturated side chains pendent from the fully saturated backbone, (Nagdi, 1993). The diene portion of the polymer chain provides pendent olefin groups as cross linking sites, (Loadman, 2005). Greater effects of degradation can be seen at the edges of the graphs concerning SBR in solutions with monochloramines than can be seen for Natural Rubber. This may be accounted for by the benzene ring found in SBR. The presence of the large ring structure may help block invasive fluids from entering into the interior of the sample having the effect of concentrating them at the outer edges of the sample, causing higher levels of degradation. As in the graphs of volume change for SBR it appears that at a point about half way through the testing cycle SBR sample begins to loose its ability to block out the fluids and rapidly looses resistance to the invasive fluids. Images of the 20 
day SBR sample are shown in Figure 3.14, where severe degradation of the carbon black at the outer edge compared to the center of the sample can be seen.
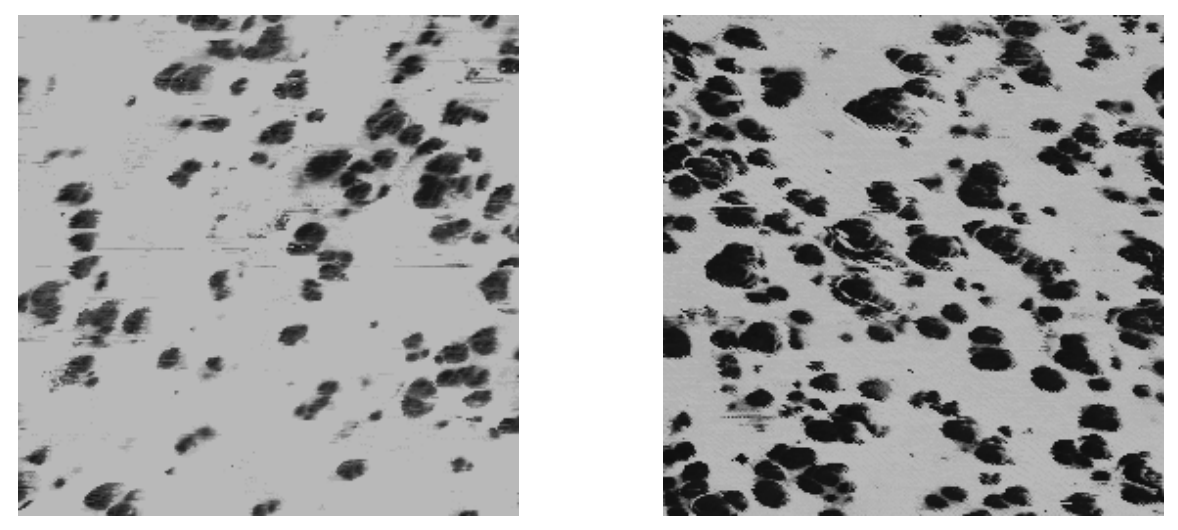

Figure 3.14 AFM images of SBR depicting effect of aging at the outer edge (left) and near the center (right) after 20 days aging with monochloramines 

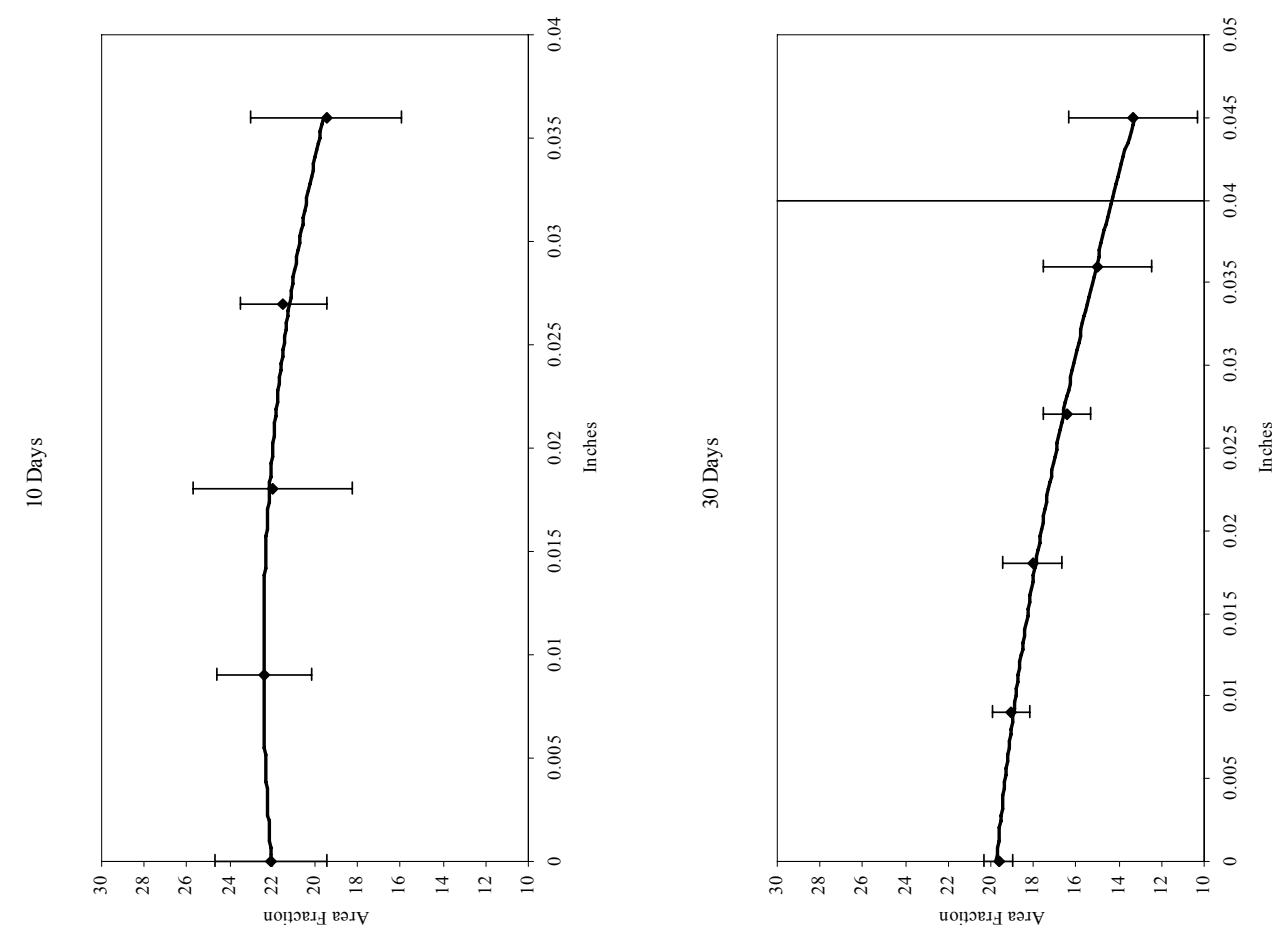

㿠
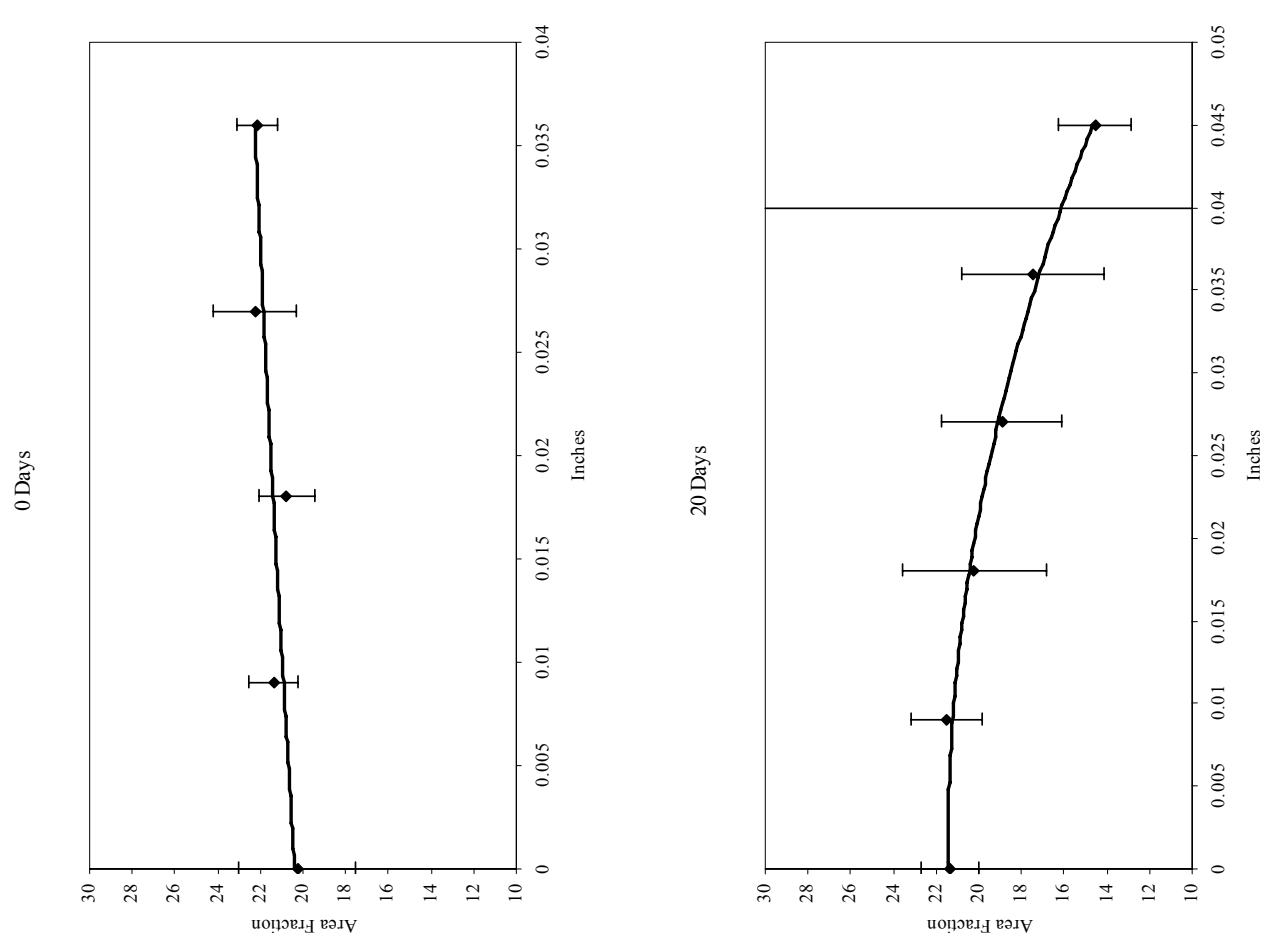

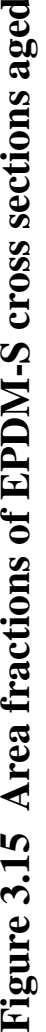



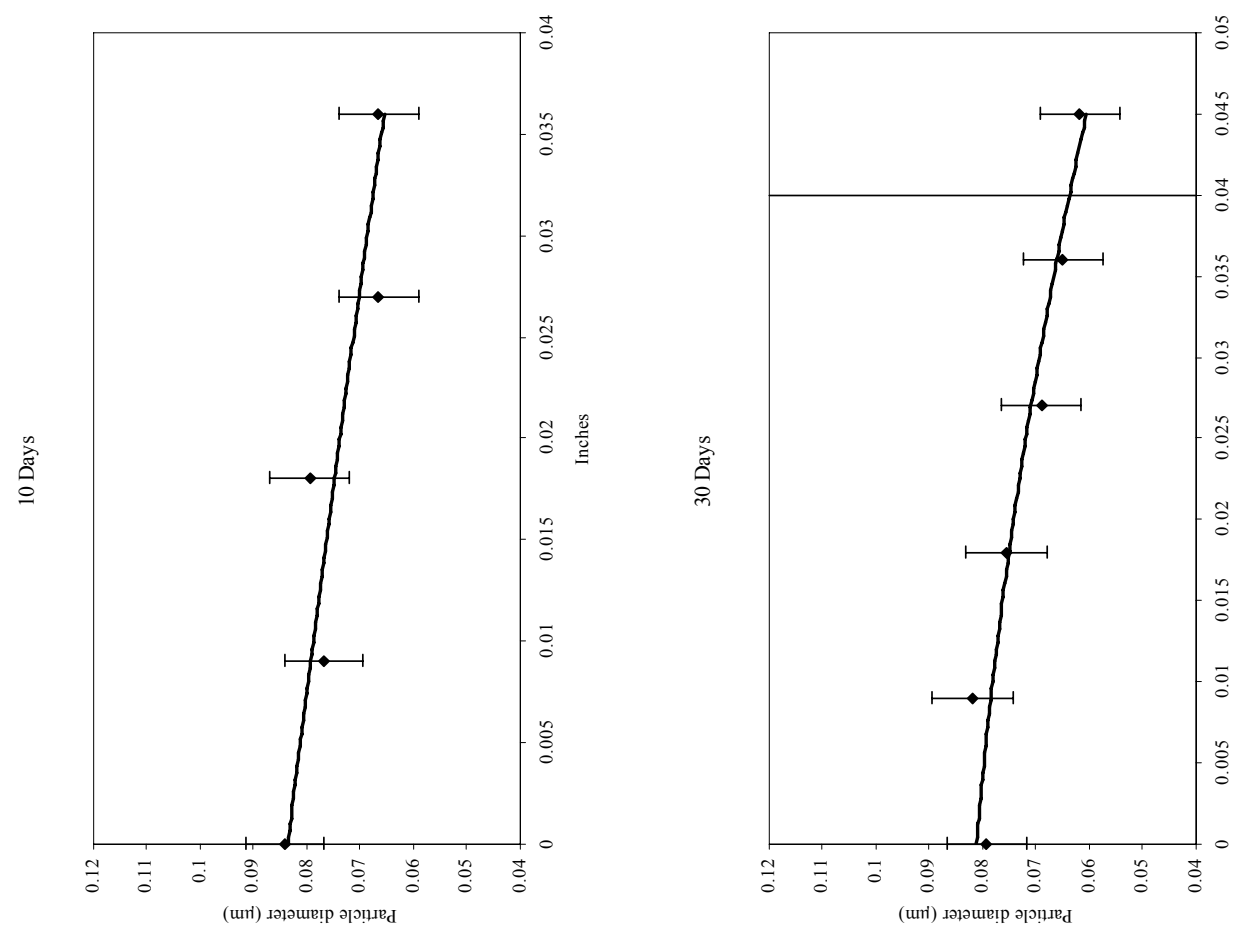

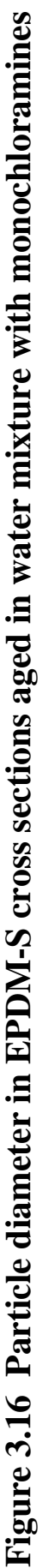
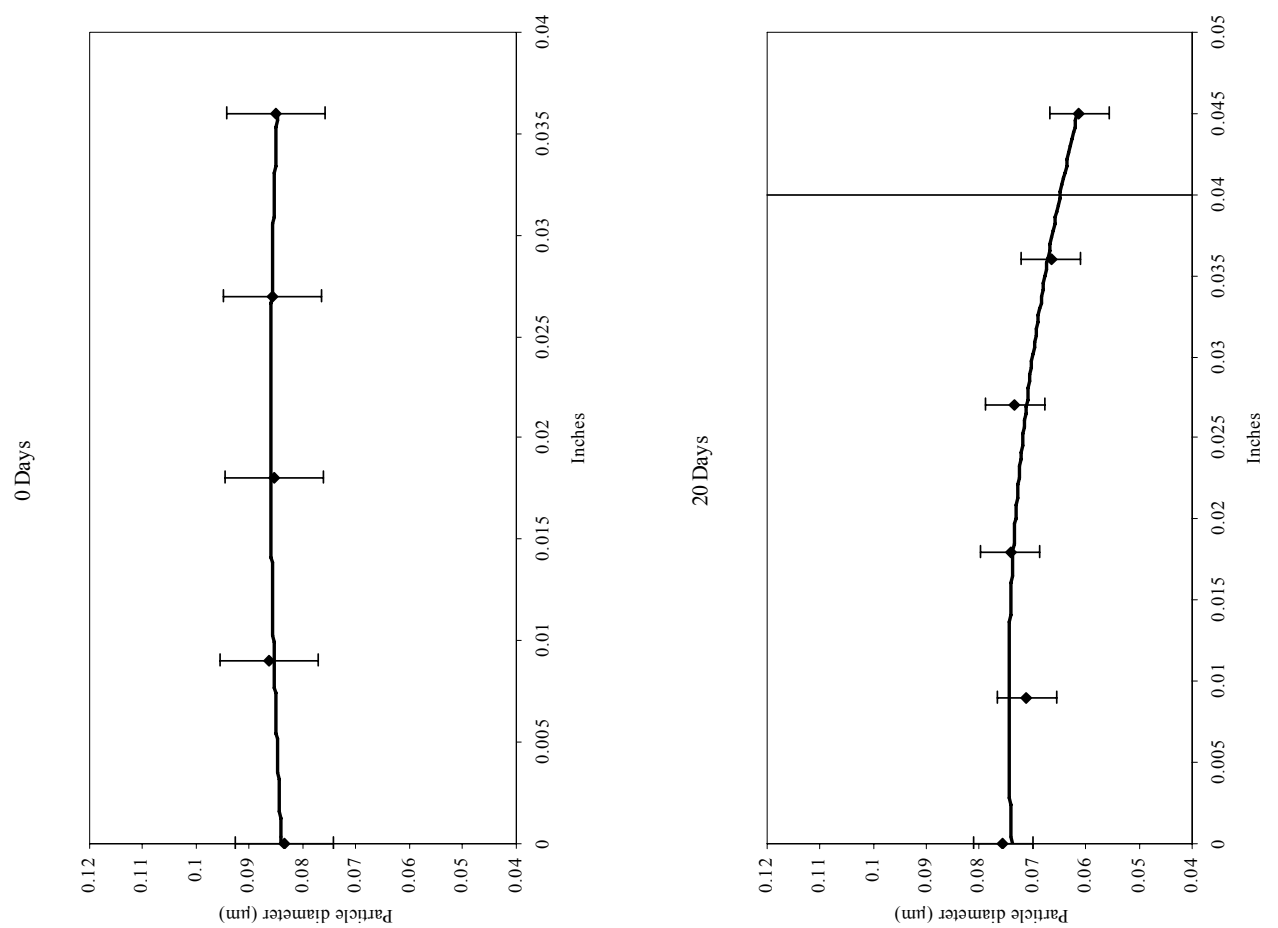
Elastomeric bulk properties during accelerated aging have been shown to degrade much more slowly for EPDM type rubbers, than for Natural Rubber or SBR. Evidence of degradation effects on carbon black particles contained within the polymer matrix showing a similar trend can be seen in Figures 3.15 and 3.16. A trendline fit to the data of area fraction covered by the carbon black at 20 days shows a still distinctly curved line. This indicates that the effects of degradation have not occurred as deeply into the sample for EPDM-S as for the other two rubber types. The same trend is true for the average particle diameter. Both aspects of degradation show a flattening of the trendline, and more consistent effects through the sample by day 30 . Figure 3.17 compares edge effects on carbon black in EPDM-S from day 0 and day 30 .
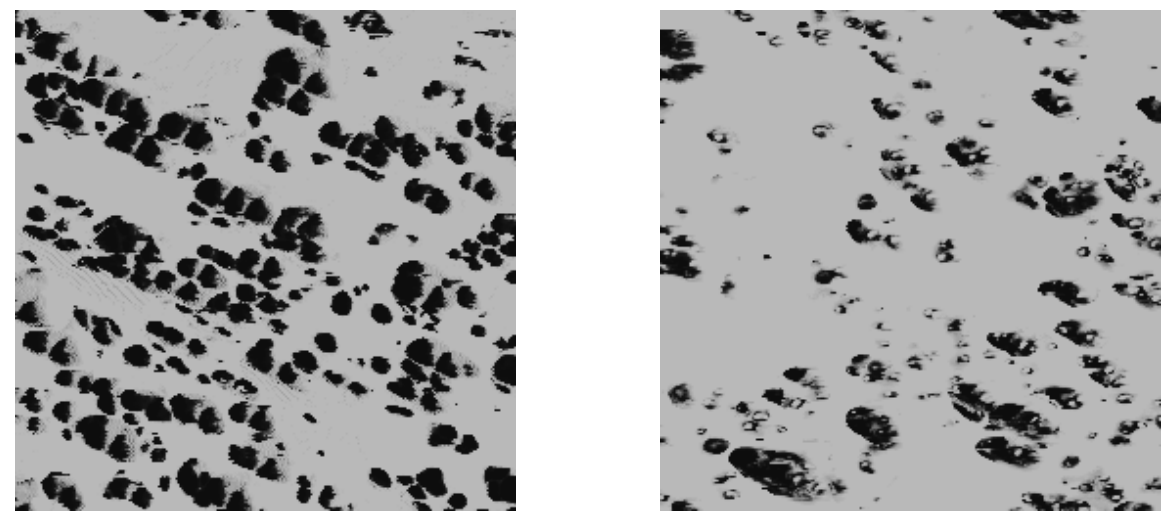

Figure 3.17 AFM images of EPDM-S depicting near edge effects at 0 days (left) and after 30 days aging with monochloramines(right) 
Decreases in area fraction and particle diameter of the three types of rubber tested coincide with changes in performance of the rubber types in conditions of accelerated aging. All three types of rubber tested show very similar trends, with EPDM-S maintaining its integrity somewhat better through 20 days of testing, but appearing to finally show similar degradation after 30 days. The 0 day graphs show an even distribution of carbon black from the middle of the sample to the outer edge. Each subsequent testing day shows that area fractions covered by the carbon black particles is decreasing beginning most dramatically at the edge. Very little loss of carbon black is detected in the middle most part of the samples. This point becomes pertinent in the derivation of Fick's second law used to estimate diffusion coefficients. Some variation in area fraction and particle diameter is due to batch variations and localized variations in the distribution of the carbon black, but in general the trends of decreasing size and area can clearly be seen to begin at the surface and gradually extend inward over time. Second degree polynomial trendlines were fit to the data to aid in visualization. In every case the area fraction appears as a flat line sloping straight downward at thirty days, indicating that the deteriorating solution has penetrated close to the center of the coupon. At 10 days the trendlines are most often curved, being near to the original values toward the center and decreasing sharply only at the outer edge. Natural Rubber and SBR show nearly flat lines of degradation after just 20 days, while EPDM-S still shows that most of the interior of the sample has not been greatly affected, thus having a trendline at 20 days that decreases sharply more toward the edge of the sample. 
Rubber coupons subjected to conditions of accelerated aging in a water mixture containing a similar concentration of chlorine and maintained at the same temperature were also analyzed using AFM techniques and selected results appear in the following figures. The results of samples soaked in mixtures with monochloramines are shown together with the chlorine results for comparison. It is evident from the data that chlorine has affected the sample in a similar manner to the mixture containing chloramines. A major difference between them is the amount of expansion of the rubber volume in the mixture with chloramines. The vertical line denoting the original sample size is hardly exceeded in the case of the chlorine samples even after 30 days. This may therefore be taken to be the relative edge of the samples. The sharply curved trendlines of the chlorine samples also indicates that erosion of the particles is almost entirely at the outer edge of the samples. This appears to coincide with the extent of diffusion into the samples interior by the penetrating fluid. In most cases the amount of erosion of the carbon black particles is greater by solution containing monochloramines than by those containing chlorine. Error bars have been included in the data pertaining to chlorine but have not been repeated as shown in earlier figures for the case of monochloramines. 

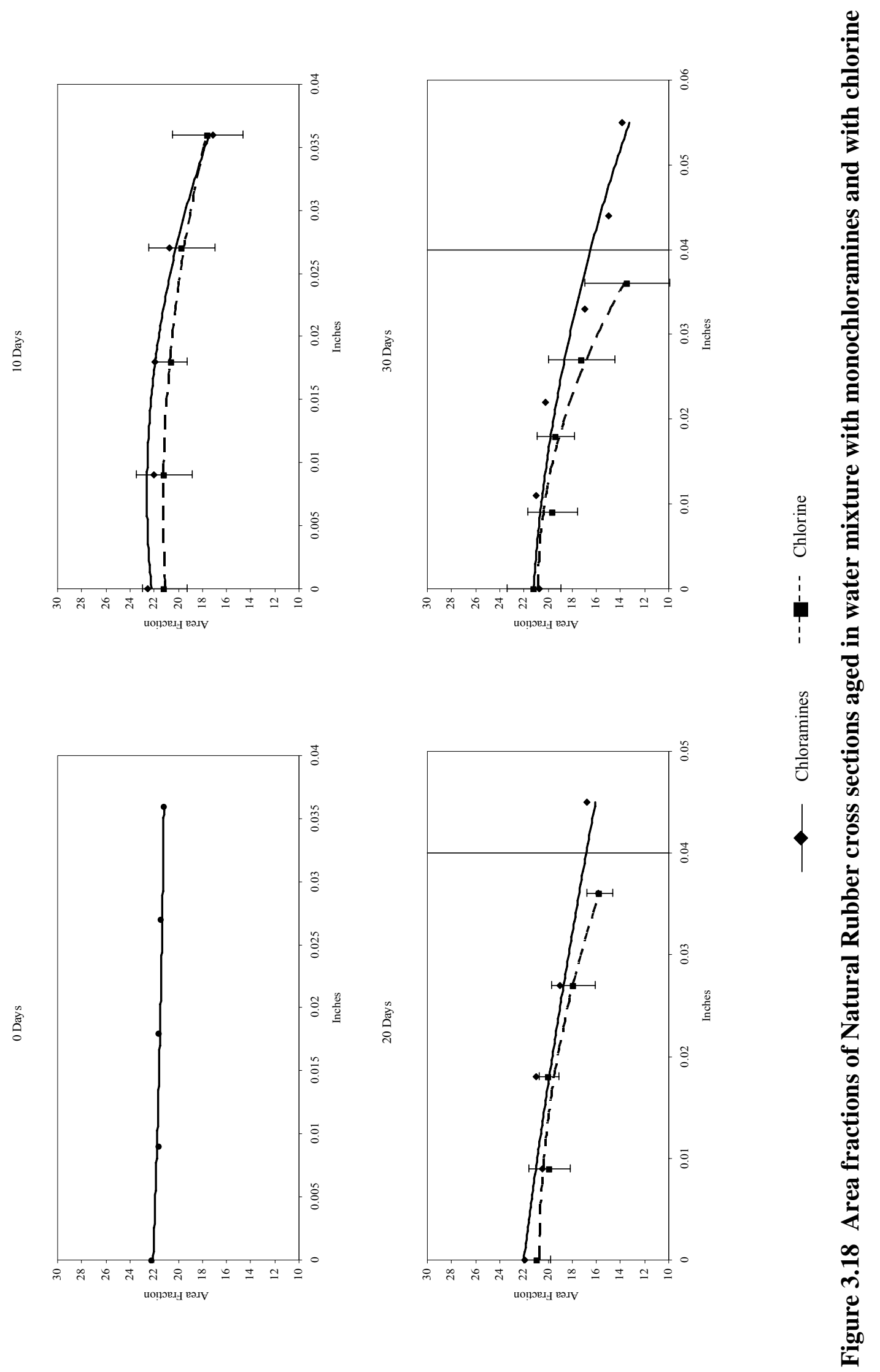

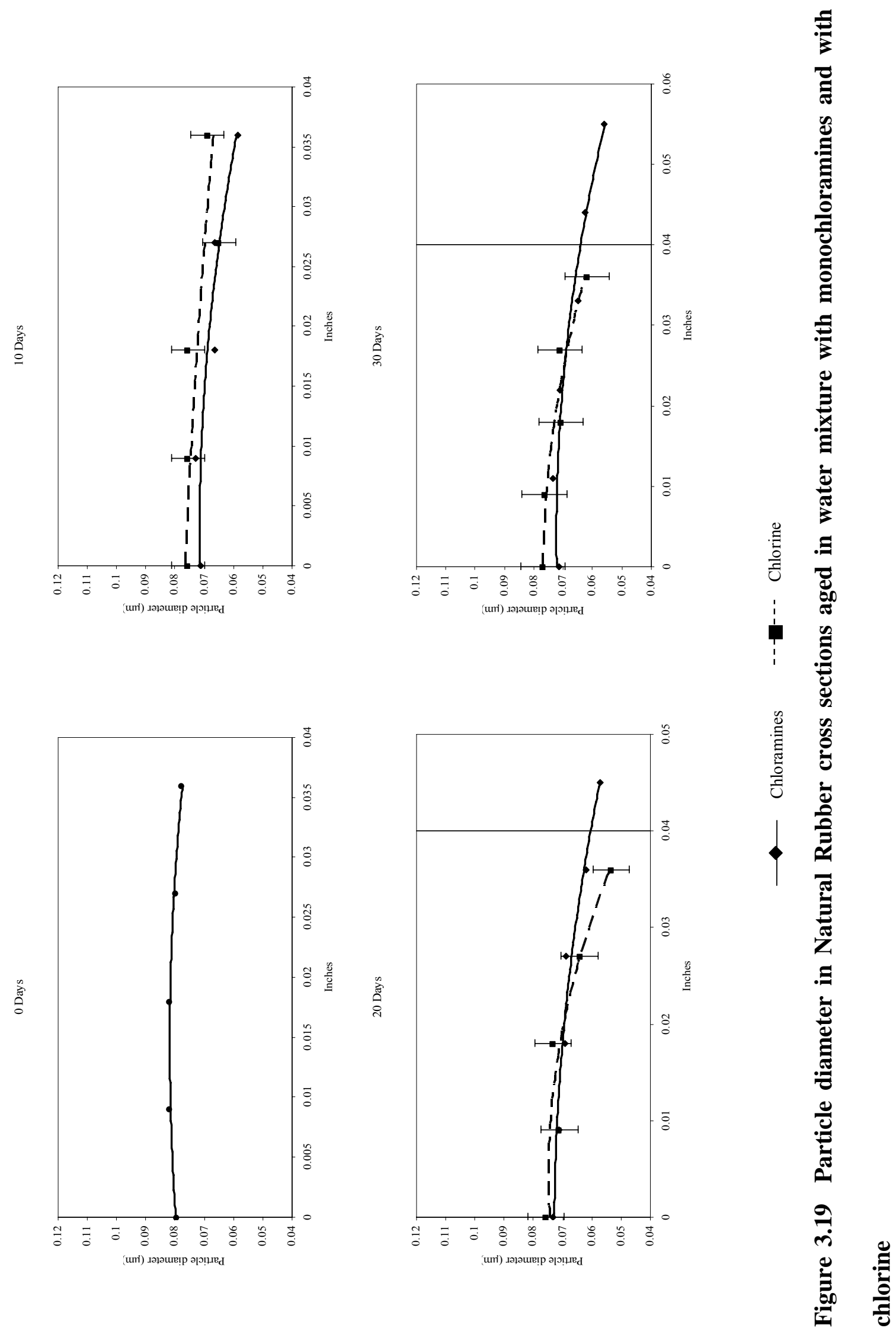

을 

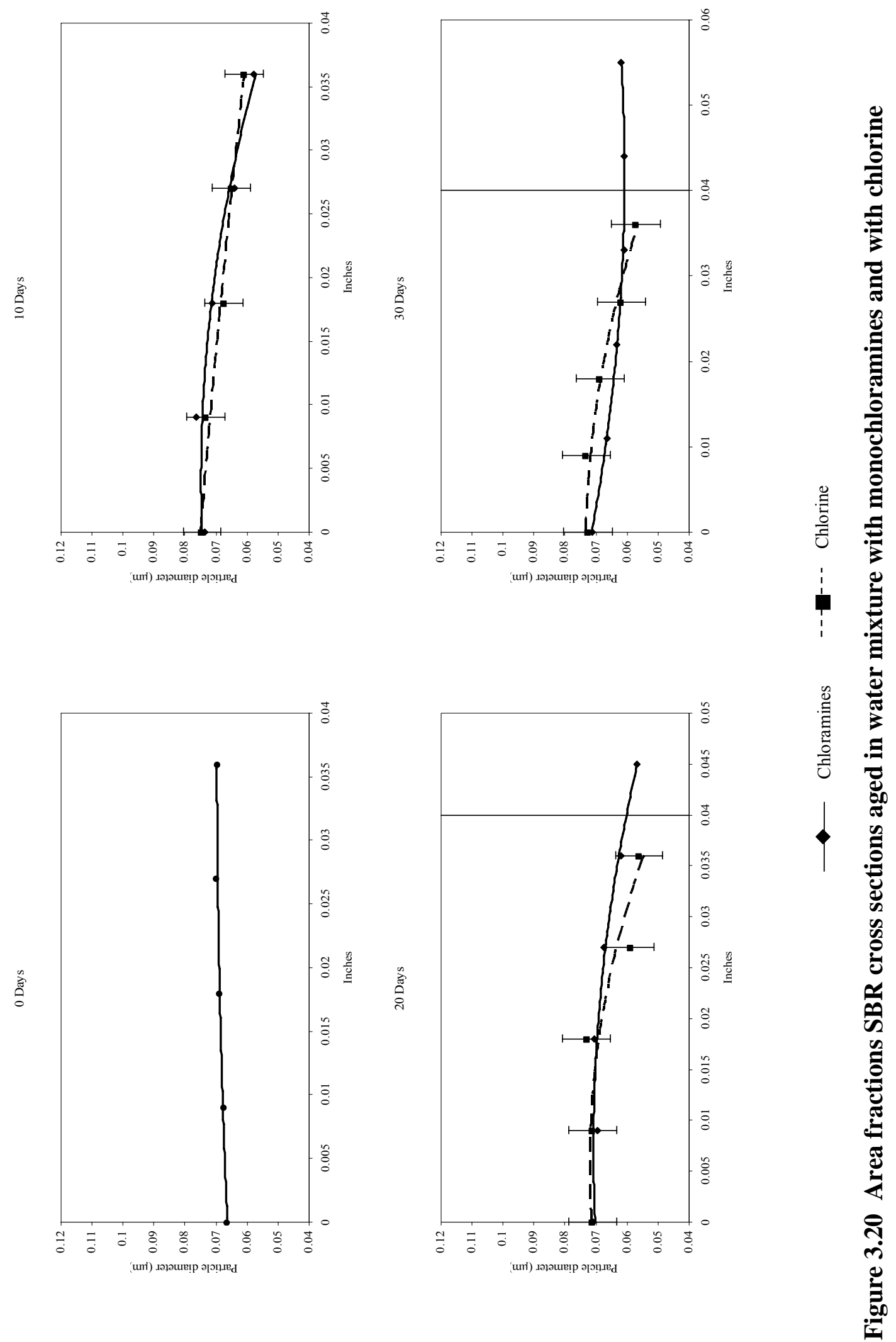

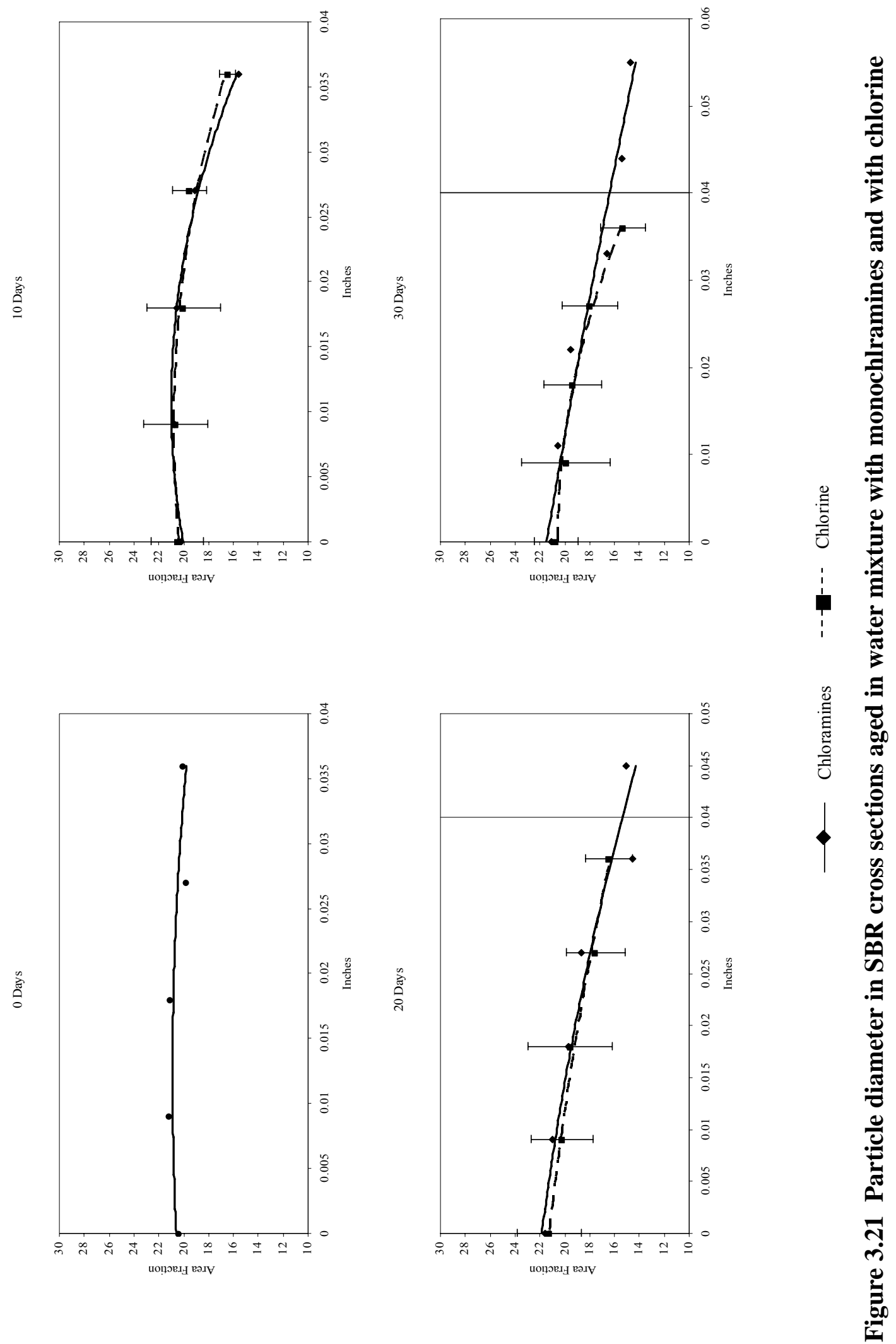

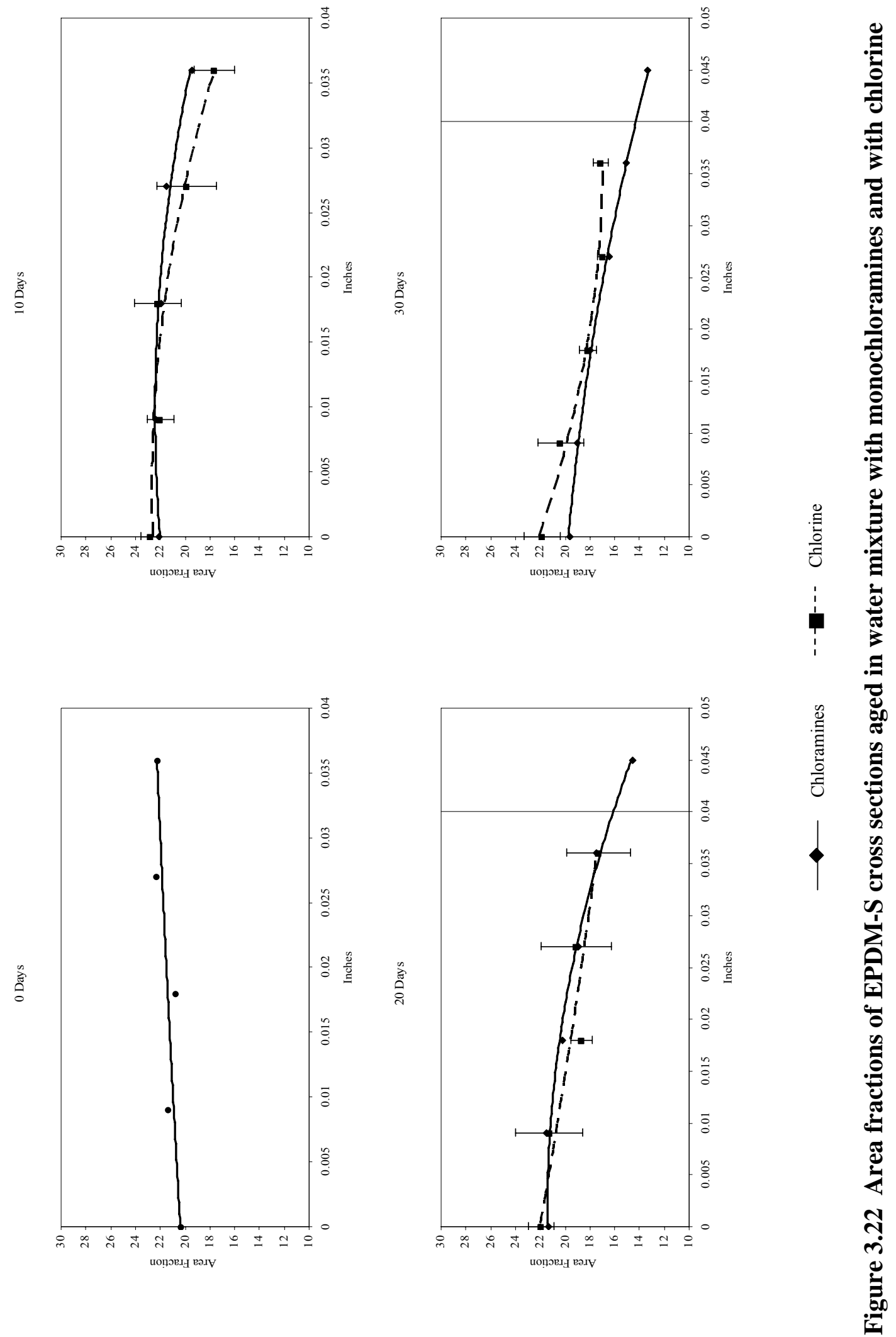

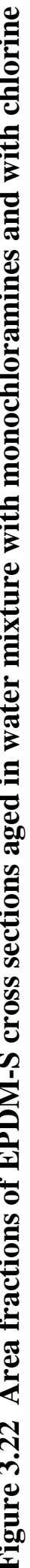



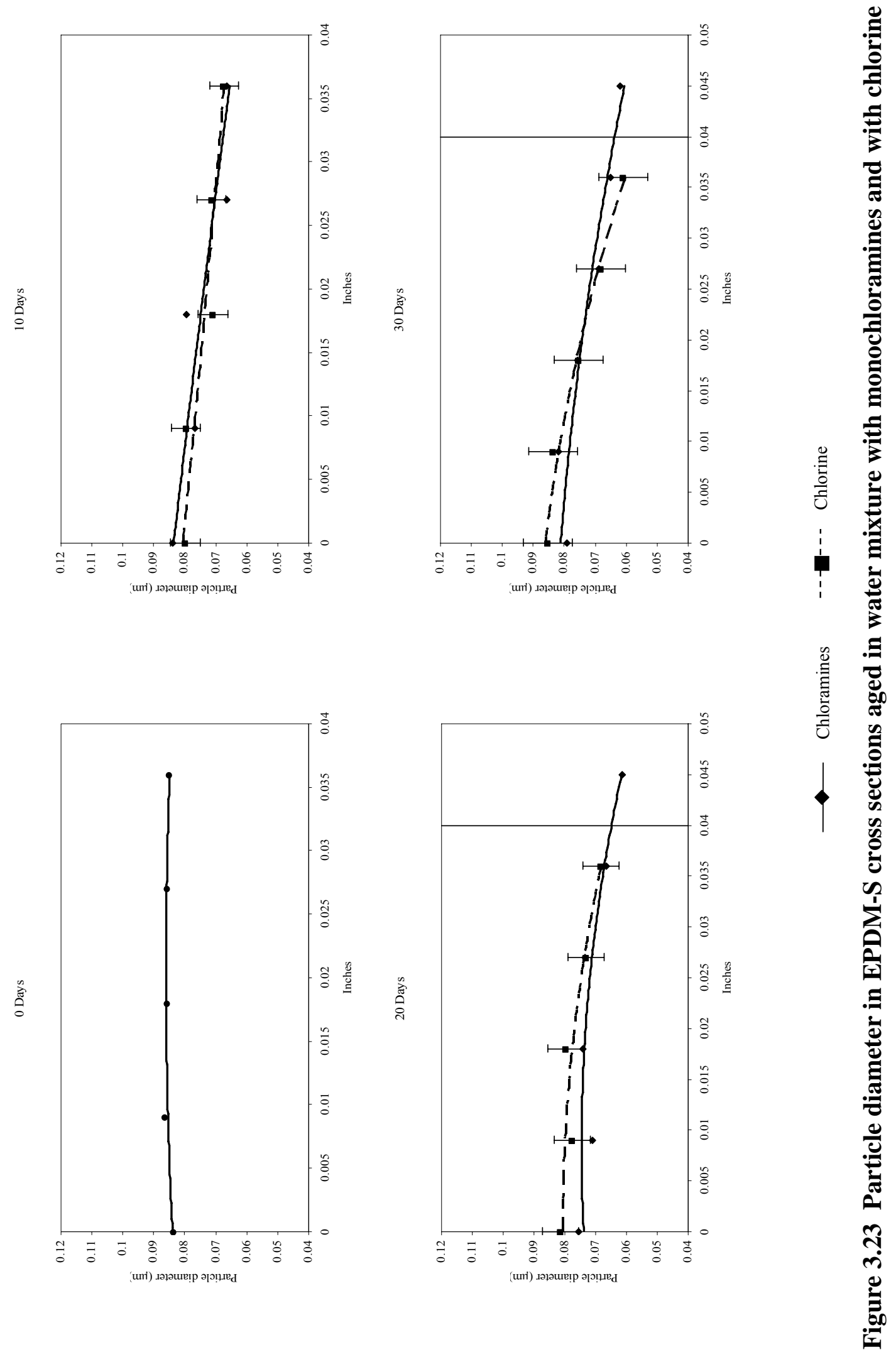

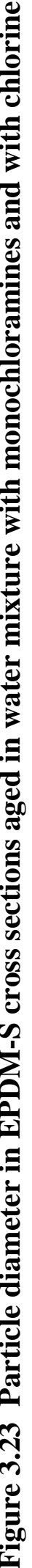


In some cases the trendlines for the samples soaked in chlorine appear to show a greater amount of degradation than for those samples aged with chloramines; such is the case for the area fraction graphs of Natural Rubber after 10 days of aging in Figure 3.18. The same is true for particle diameter of EPSM-S after 10 days accelerated aging as shown in Figure 3.21. The apparent more severe degradation of these samples due to chlorine is thought to be indicative of variations in batch mixtures and somewhat due to limitations and errors involved with imaging and analysis. Micrographs of typical AFM images of samples aged in a chlorine environment are shown in Figure 3.24, the sample depicted is Natural Rubber and are similar to images taken of EPDM-S and SBR upon aging in a chlorine environment.
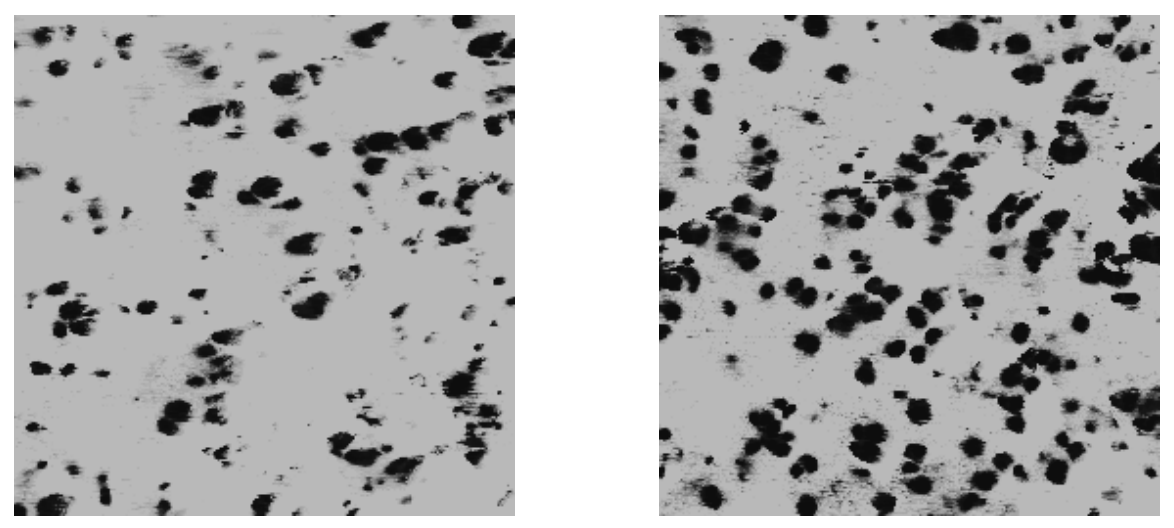

Figure 3.24 AFM images of Natural Rubber depicting effect of aging at the outer edge (left) and near the center (right) after 30 days with chlorine 


\section{Estimated Diffusion Coefficients}

Micrographs illustrating optical microscope images of the three types of rubber tested are shown in Figure 3.25. The different rates of diffusion of water mixed with disinfectants are a point of interest in research into the effects that monochloramines have on the rubber sample as compared to chlorine. Each of the micrographs depicts a cross sectioned sample of the 0 day material to the left, 30 days accelerated aging in water containing chlorine in the center and after 30 days aging with monochloramines to the right. The zone of expansion at the edges of the specimens can clearly be seen in samples aged with chloramines.
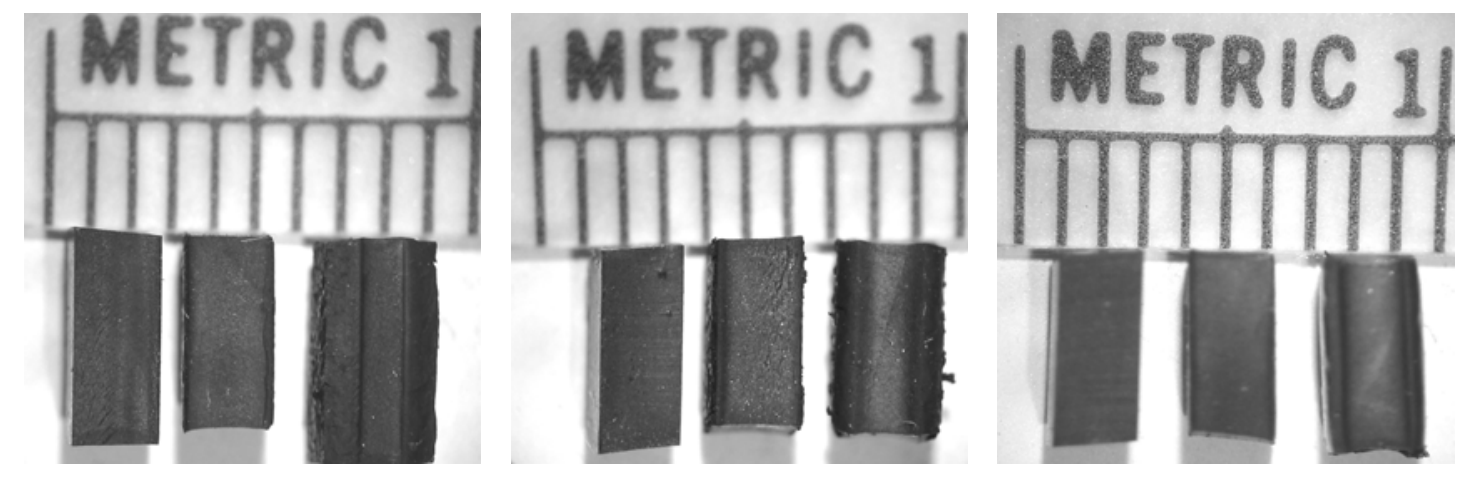

Figure 3.25 Sample Cross Sections at 0 days (left), 30 days in chlorine mixture (center) and monochloramines mixture (right) of each individual micrograph with Natural Rubber shown first, SBR second and EPDM-S third

Zones of expansion appear as raised or bulbous ridges at the sides of the sample. In samples where accelerated aging was accomplished in a mixture containing chlorine 
the ridges are hardly noticeable and do not extend very far into the sample. The depth of the ridges coincides well with the AFM data shown above. The loss of area fraction and decrease in particle diameter extending from the edge into the interior of the samples appears similar to the depth of the ridges viewed under an optical microscope. The effective rate of diffusion for invasive fluids into each of the three types of rubber for both monochloramines and chlorine mixtures has been calculated using a simplified form of Fick's second law. Derivation of the simplified equation is described in Schaffer et al., (1999).

We can write Equation 1, describing the rate of change of the number of particles of any substance in a geometric volume where $\mathrm{J}$ is the flux in and out of a thin slice of the volume, where $\mathrm{C}$ is the concentration, $\mathrm{t}$ is the time, $\mathrm{V}$ is the volume and $\mathrm{A}$ is the area of the thin slice.

$$
\left(\frac{\partial C}{\partial t}\right) d V=\left(J_{\text {in }}-J_{\text {out }}\right) d A
$$

Recognizing that $d \mathrm{~V}=d \mathrm{~A} d \mathrm{x}$ and rearranging yields Equation 2 .

$$
\frac{\partial C}{\partial t}=\frac{J_{\text {in }}-J_{\text {out }}}{\partial x}=-\frac{\partial J}{\partial x}
$$

Ficks first law is given as Equation 3. 


$$
J=-D\left(\frac{d C}{d x}\right)
$$

By substituting the expression for flux in Equation 3 we arrive at Equation 4.

$$
\frac{\partial C}{\partial t}=D\left(\frac{\partial^{2} C}{\partial x^{2}}\right)
$$

Under conditions of a continuously replenished source the solution yields Equation 5.

$$
\frac{C(x, t)-C_{0}}{C_{S}-C_{0}}=1-\operatorname{erf}\left(\frac{x}{2 \sqrt{D t}}\right)
$$

The concentration at the effective penetration distance can be estimated using Equation 6 .

$$
C\left(x_{\text {eff }}, t\right)=\frac{C_{0}+C_{S}}{2}
$$

By substituting this value into Equation 5 the result yields Equation 7.

$$
0.5=\operatorname{erf}\left(\frac{x_{\text {eff }}}{2 \sqrt{D t}}\right)
$$

Since the error function erf $(0.5)$, can be shown to approximately equal to 0.5 , then we therefore arrive at the simplified version of Fick's second law used to estimate diffusion coefficients given in Equation 8. 


$$
x_{\text {eff }} \approx \sqrt{D t}
$$

The simplified form of the equation assumes a semi-infinite distance where permeates moving in from the sides do not meet. As Figure 3.25 shows liquid diffusing in from the sides of the rubber coupon has not effectively reached the center. This assumption is reinforced by AFM data which shows very little loss of carbon black in the middle of the samples. Therefore the supposition of a semi-infinite distance is upheld. The effective penetration distance is defined as the point at which the concentration of the diffusing species has an equal value to the average of the concentration at the surface and the initial concentration within the sample (Schaffer et al., 1999). In this analysis the effective penetration distance is given by the clearly discernable zone of expansion at the edges of the sample measured with digital calipers.

During the accelerated aging cycle all six faces of the rubber coupon are expanding outward at the same rate, as swelling of the interior of the sample takes place. The distance has therefore been estimated using an average between the distance of the original sample and the inflated distance. Volume of the coupon is given by the product of the three dimensions. In spite of the geometric expansion of the rubber in all three dimensions it was concluded that a straight arithmetic average could be used rather than a geometric weighted average. Since the dimensions of the coupon are such that the length and width are much greater than the thickness, expansion of the volume in the longest dimension can be assumed negligible. A substitution may then be made describing the 
area of the expanding slice giving an estimated change in area equal to the product of (x $+\Delta \mathrm{x})^{2}$. Since the result is a polynomial equation such an equation has been fit to the data concerning volume change and a representative example has been shown in Figure 3.26. In order to show general trends of carbon black degradation a second degree polynomial trendline was fit to each of the graphs resulting from the AFM data. In Figure $3.26 \mathrm{R}^{2}$ values have been displayed for each of the second degree polynomial trendlines. If the assumptions of expansion hold true then the $\mathrm{R}^{2}$ value should be close to one.

\section{Volume Change}

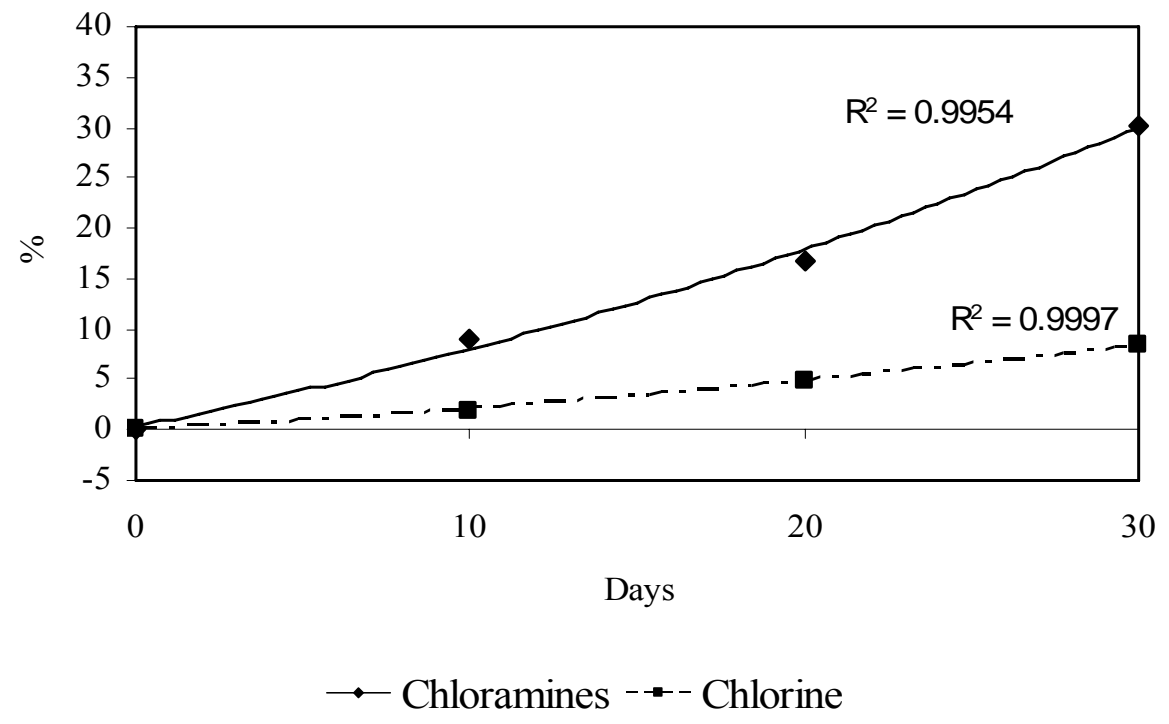

Figure 3.26 Volume data with trendlines and $\mathbf{R}^{2}$ values for EPDM-S

The $\mathrm{R}^{2}$ values shown in Figure 6.26 are the worst case of the three types of rubber tested and are sufficiently close to one for the purpose of estimating diffusion coefficients. Several distance measurements were made for each sample the deviation in 
measurements due to mechanical accuracy of the calipers and uneven distribution within the samples has been used to estimate error in the diffusion coefficients, shown in Table 3.1. The diffusion coefficients are directly proportional to the rate at which diffusion occurs into and out of the sample. Results of estimating the diffusion coefficients can be seen in Table 3.1 .

Table 3.1

Diffusion coefficients determined after accelerated aging for 30 days

\begin{tabular}{cccc}
\hline & $\begin{array}{c}\text { Natural Rubber } \\
\left(\mathrm{cm}^{2} / \mathrm{sec}\right)\end{array}$ & $\begin{array}{c}\text { SBR } \\
\left(\mathrm{cm}^{2} / \mathrm{sec}\right)\end{array}$ & $\begin{array}{c}\text { EPDM-S } \\
\left(\mathrm{cm}^{2} / \mathrm{sec}\right)\end{array}$ \\
\hline Chloramines & $1.42 \mathrm{E}-9 \pm 1.2 \mathrm{E}-10$ & $1.26 \mathrm{E}-9 \pm 1.8 \mathrm{E}-10$ & $3.70 \mathrm{E}-10 \pm 3.0 \mathrm{E}-11$ \\
Chlorine & $3.19 \mathrm{E}-11 \pm 1.3 \mathrm{E}-11$ & $2.40 \mathrm{E}-11 \pm 1.1 \mathrm{E}-11$ & $1.03 \mathrm{E}-12 \pm 8.3 \mathrm{E}-13$
\end{tabular}

As expected similarities of Natural Rubber and SBR persist in the diffusion rates as for carbon black degradation and elastomeric properties. Diffusion coefficients for the two types of rubber are of the same order of magnitude in the case of both monochloramines and chlorine. In the case of EPDM-S the diffusion rate and therefore the estimated diffusion coefficient is much less, this is possibly due to the crystalline structure of the material. Diffusion coefficients for SBR are slightly smaller than for Natural Rubber. This may be explained by the large ring structure present in the polymer chain of SBR as compared to a corresponding methyl group present in Natural Rubber. Monochloramines show a much greater effect on the rubber than does the use of chlorine as disinfectants. The order of magnitude of the diffusion coefficients are less for chlorine than for monochloramines in every case. Table 3.2 compares the values of diffusion 
coefficients estimated in this report using the simplified form of Fick's second law for Natural Rubber and SBR in disinfectant water using monochloramines to those estimated usingr basic diffusion equations proposed by Crank, J. (1975). This solution has two equations one for short term and other for long term. Balik C.M. (1996), proposed a combined equation with appropriate weight factors for short term and long term equations. (Nagisetty, 2007)

Table 3.2

\section{Comparison of estimated diffusion coefficients of solution with chloramines}

\begin{tabular}{ccc}
\hline Method & $\begin{array}{c}\text { Natural Rubber } \\
\left(\mathrm{cm}^{2} / \mathrm{sec}\right)\end{array}$ & $\begin{array}{c}\text { SBR } \\
\left(\mathrm{cm}^{2} / \mathrm{sec}\right)\end{array}$ \\
\hline Simplified Fick's & $1.42 \mathrm{E}-9 \pm 1.2 \mathrm{E}-10$ & $1.26 \mathrm{E}-9 \pm 1.8 \mathrm{E}-10$ \\
Nagisetty, 2007 & $1.38 \mathrm{E}-09$ & $1.85 \mathrm{E}-09$
\end{tabular}

Comparison of the diffusion coefficients shows a high level of agreement between the two methods of estimation. The analytical approach given by Nagisetty (2007), not only agree in order of magnitude but are only a few percentage points different than the values estimated in this report using effective penetration distance determined by measurement of the highly saturated regions around the perimeter of the samples. Monochloramines have been found to have a greater impact on the degradation of carbon black in a similar manner to the degradation of the materials elastomeric properties as well as the diffusion coefficients. 


\section{Conclusions and Recommendations}

Data procured during this experiment are representative of typical results seen in earlier experiments concerning performance of Natural Rubber, SBR and EPDM-S under conditions of accelerated aging. Tensile testing of maximum stress and strain at elongation was not done in this experiment though other elastomeric properties of the bulk material such as changes in hardness, mass and volume of the samples were. Data from this experiment matched earlier experimentation results well and it can be assumed that tensile testing would also mirror the results of prior testing. In previous studies it has been found that testing of tensile strength shows similar trends to the other elastomeric properties, the maximum stress and strain values decreased much more rapidly when exposed to mixtures with monochloramines than with chlorine with EPDM-S retaining its integrity much better than Natural Rubber and SBR which where similar. As has been stated earlier the rate of diffusion of the penetrating fluid into the sample is likely to be responsible for the loss of carbon black which in turn contributes to the deterioration of the bulk properties of the rubber. It may also be assumed that the tensile properties are dependant upon the relative rates of diffusion. Material at the perimeter of the samples expands in clearly discernable zones. The soft material in these expanded zones shows a 
much greater loss of carbon black material than in the middle of the sample. It is likely therefore that the deterioration of the bulk properties is due mainly to carbon black losses in the expanded regions and that tensile strength is dependant on the continuously shrinking region in the middle which appears far less affected by the penetrating fluid.

AFM imaging of the samples exposed to conditions of accelerated aging has shown an overall loss of carbon black material and a decrease in the average particle size. Degradation of the carbon black coincides with deterioration of bulk properties and occurs in an increasing region around the perimeter of the sample similar to the zones of expansion. These results, along with earlier observations based on the turbidity of the extracted fluids, strongly suggest that the monochloramines have induced erosion of the carbon black particles which is a strong factor in the decrease in rubber performance over time. Evidence from the analysis of AFM data shows that carbon black is eroded away gradually over time by the disinfectant solutions penetrating into the sample. It does not appear that carbon black leaves the sample as particles of the size originally found within the polymer matrix.

Atomic Force Microscopy has shown trends in carbon black degradation of both size and number. A greater quantity of data would be useful to fully characterize the effect of monochloramines on the carbon black. Due to mechanical issues of the AFM it becomes more difficult to produce meaningful phase images of the particles as the polymer matrix degrades. Solutions to this problem could be to use coated AFM tips as well as the use of tips with more beneficial characteristics. One such characteristic could 
be the use of sharper tips with a nominal radius of less than 10 nanometers, such as the tip used in this experiment. A sharper tip would be capable of more accurately defining the shape and size of smaller particles since the lower limit of the particle size which can be imaged is approximately equal to the size of the tip. Coating of the tip could also improve image quality in order to impart a more hydrophobic characteristic onto the surface of the tip. This would help to eliminate some of the data scatter caused by capillary action that forms between the tip and the surface, thereby creating sharper images. Since the tips used in this experiment are etched from silicon there is a thin silicon oxide layer on the tip. The use of silane chemistry to modify the tip can produce a tip coated with trichlorosilane molecules. The hydrophobic nature of the surface coating would be very beneficial for imaging the surface of a polymer which may have surface moisture. A tip with a resonance frequency that mimics surface conditions of hardness or elastic modulus would improve the ability of the AFM to discern between physical properties. An ideal cantilever would have a spring constant that relates to an elastic modulus that lies between the modulus of the two materials such as the hard carbon black and the soft polymer matrix.

A Nova 600 SEM was used for the purposes of this experiment. Gold coating which can lead to certain features being washed out was used in imaging day 0 samples. Coating the sample surface was necessary due to the non-conductive nature of the rubber. The elastomer sample may be imaged by using lower power of the electron beam. A lower power SEM would have less chance of burning the sample. The problem of saturation would still prevent use of the SEM due to expansive gasses out of the sample. 
The environmental SEM that was used for the purposes of this experiment was supplied with a sample cell capable of imaging in liquid but not completely submerged. A silicon nitride capsule that contains both the sample and liquid of choice may be obtained. This would allow imaging of the saturated sample in the environment of use for the rubber. Some loss of resolution possibly occurs from the use of the silicon nitride capsule but may provide clear enough images to be useful. Imaging the rubber while maintaining the liquid environment in which it has aged would prevent changes from drying and shrinking during imaging. The SEM images can be analyzed using software such as imageJ in the same manner as the AFM. A greater quantity of data produced by SEM methodologies or AFM reaffirming the findings of this study are necessary to understand more completely the degrading effects that monochloramines have on rubber parts, particularly the possible erosion and washing out of the carbon black material. Monochloramines use as a disinfectant agent by the water utility industry has proven to degrade rubber parts by modes which include deterioration of the filler material carbon black at a faster rate than the use of chlorine. Despite this consequence monochloramines have a longer term of effectiveness as a disinfectant and produce fewer toxic by-products. Suitable rubber formulations which show resistance to attack by monochloramines should be further examined. 


\section{REFERENCES CITED}

Balik C.M., 1996. On the extraction of diffusion coefficients from gravimetric data for sorption of small molecules by polymer thin films, Macromolecules, 29(8), 3025-3029.

Binnig, G., C. F. Quate and C. Gerber, 1986. Atomic force microscope Physics Review Letter 56:930-933.

Crank, J., The Mathematics of Diffusion ( $2^{\text {nd }}$ ed), Oxford University Press, New York, Page 48, (1975).

Chernoff, D. A., Proc. Microscopy and Microanalysis (Bailey, G. W., Ellisman, M. H., Hennigar, R. A., Zaluzec, N. J., Eds), 1995. New York: Johnes and Begell Publishing.

Donnet, J-B. and A. Voet, Carbon Black Physics Chemistry and Elastomer

Reinforcement, Mercel Dekker, Inc., New York, Pages 51 and 297, (1976).

Kwak, S.K., G.S. Lee, D.J. Ahn and J.W. Choi, 2004. Pattern Formation of cytochrome c by microcontact printing and dip-pen nanolithography. Material science and Engineering 24:151-155.

Loadman, John, Tears Of The Tree, Oxford University Press, New York, Page 182, (2005).

Martin, Y., C.C. Williams and H.K. Wickramasinghe, 1987. Atomic force microscopeforce ampping and profiling on a sub 100-A scale, Journal of applied Physics 61(10):4723-4729.

Nagdi, Khairi, Rubber as an Engineering Material, Carl Hanser Verlag, New York, Page 110, (1993).

Nagisetty, R. M., R. Schoenbaechler, T. Rockaway and G. Willing, 2007. Paper in preparation for publication.

Osswald, Tim A., Georg Menges, Materials Science of Polymers for Engineers, Hanser Gardner Publications, Inc., Ohio, Page 5, (2003).

Reiber, S., 1993. Investigating the Effects of Chloramine on Elastomer Degradation. Journal of the American Water Works Association. 85:8:101.

Rockaway, Thomas D., Gerold A. Willing, Raja Mohan Nagisetty, 2007. Life Prediction of Elastomers in Drinking Water Distribution Systems Journal of the American Water Works Association Accepted. 
Rugar, D. and P. Hansam, 1990. Atomic force microscopy. Physics Today 43:23-30.

Snoeyink, Vernon L. and David Jenkins, 1993. Water Chemistry. John Wiley and Sons.

Schaffer, J. P., A. Saxena, S. D. Antolovich, T. H. Sanders Jr. and S. B. Warner, The Science and Design of Engineering Materials ( $2^{\text {nd }} e d$ ), McGraw-Hill, New York, Pages 139 and 601, (1999).

Schreck, M., 2006. Private Communication.

Valleru, Jahnavi, 2006. Kinetics of Sulfur and Peroxide Cured EPDM Rubber Aging in Chloraminated Water. Master of Engineering thesis, University of Louisville.

Willing, Gerold A., 2007. Private Communication.

Yang, J., K. Takeyasu, and Z. Shao, 1992. Atomic force microscopy of DNA molecule. FEBS Letters 301(2):173-176. 
Randolph Norman Schoenbaechler was born on December 1, 1972 in Louisville, Kentucky. He attended Trinity High School in Louisville and graduated in 1990. After high school he earned a Bachelor of Science with a concentration in Biotechnology and a Minor in Philosophy at the University of Louisville in 1999. After working in the civil engineering field he was accepted in the University of Louisville Speed Scientific School in the summer of 2002.

He was treasurer of the Graduate Student Association, and a member of the American Institute of Chemical Engineers. He completed three semesters of cooperative education by working at a pigment manufacturing plant in Louisville.

He received his Bachelors of Science in Chemical Engineering in the spring of 2005 and completed his Masters of Engineering in Chemical Engineering with Environmental Certificate in the summer of 2007. 